\title{
General Syntheses of Nanotubes Induced by Block Copolymer Self-Assembly
}

Zhao, Jianming; Huang, Wei; Si, Pengchao; Ulstrup, Jens; Diao, Fangyuan; Zhang, Jingdong

Published in:

Macromolecular Rapid Communications

Link to article, DOI:

10.1002/marc.201800125

Publication date:

2018

Document Version

Peer reviewed version

Link back to DTU Orbit

Citation (APA):

Zhao, J., Huang, W., Si, P., Ulstrup, J., Diao, F., \& Zhang, J. (2018). General Syntheses of Nanotubes Induced by Block Copolymer Self-Assembly. Macromolecular Rapid Communications, 39(12), e1800125.

https://doi.org/10.1002/marc.201800125

\section{General rights}

Copyright and moral rights for the publications made accessible in the public portal are retained by the authors and/or other copyright owners and it is a condition of accessing publications that users recognise and abide by the legal requirements associated with these rights.

- Users may download and print one copy of any publication from the public portal for the purpose of private study or research.

- You may not further distribute the material or use it for any profit-making activity or commercial gain

- You may freely distribute the URL identifying the publication in the public portal

If you believe that this document breaches copyright please contact us providing details, and we will remove access to the work immediately and investigate your claim 
DOI: $10.1002 /$ marc.

\section{Communication}

\section{General Syntheses of Nanotubes Induced by Block Copolymer Self-Assembly}

Jianming Zhao, Wei Huang, Pengchao Si, Jens Ulstrup, Fangyuan Diao and Jingdong Zhang*

Dr Jianming Zhao, Wei Huang, Prof. Jens Ulstrup, Prof. Jingdong Zhang

Department of Chemistry, Technical University of Denmark, Kemitorvet 207, DK-2800

Kongens Lyngby, Denmark.

E-mail: jz@kemi.dtu.dk

Prof. Pengchao Si, Fangyuan Diao

Key Laboratory for Liquid-Solid Structural Evolution and Processing of Materials, Ministry of Education, School of Materials Science and Engineering, Shandong University, Jinan 250061, People's Republic of China.

Amphiphilic block copolymer templating strategies have been extensively used for syntheses of mesoporous materials. However, monodisperse tubular nanostructures are limited. Here, a general method is developed to synthesize monodisperse nanotubes with narrow diameter distribution induced by self-assembly of block copolymer. 3-Aminophenol (AP) and formaldehyde (F) polymerize and self-assemble with cylindrical PS-b-PEO micelles into worm-like PS-b-PEO@APF composites with uniform diameter $(49 \pm 3 \mathrm{~nm})$. After template extraction, worm-like APF polymer nanotubes were formed. The structure and morphology of polymer nanotubes can be tuned by regulating the synthesis conditions. Furthermore, PS-bPEO@APF composites are uniformly converted to isomorphic carbon nanotubes with large surface area of $662 \mathrm{~m}^{2} / \mathrm{g}$, abundant hierarchical porous frameworks and nitrogen doping. Meanwhile, the synthesis can be extended to silica nanotubes. These findings open an avenue to design of porous materials with controlled structural framework, composition and properties for a wide range of applications. 


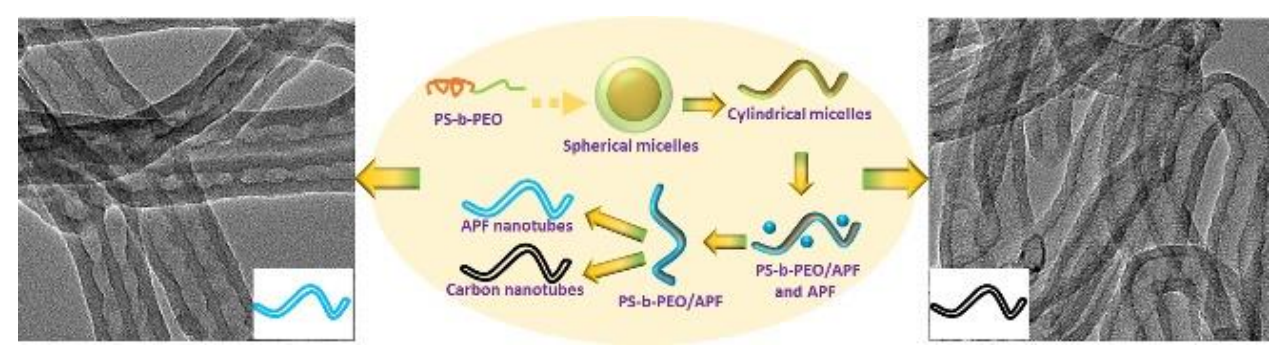




\section{Introduction}

Porous nanomaterials templated by self-assembly of amphiphilic molecules have attracted attention due to their controllable compositions, and unique structures and properties. ${ }^{[1]}$ Since the discovery of the M41S family of well-ordered mesoporous silicate/aluminosilicates in 1990s, ${ }^{[2]}$ nanoporous materials such as silica, ${ }^{[3]}$ carbon,,${ }^{[4]}$ metal, ${ }^{[5]}$ and metal oxides, ${ }^{[6]}$ have been advanced rapidly. Two main formation mechanisms have been proposed. One is liquidcrystal templating, ${ }^{[2,7]}$ in which liquid- or semi-liquid-crystal mesophases are formed during surfactant self-assembly. The other mechanism is cooperative self-assembly, ${ }^{[8]}$ based on the interactions between precursors and surfactants. Furthermore, a "silicate rod assembly" mechanism in which long silicate/surfactant rods are formed by silicate precursors depositing on isolated cylindrical surfactant micelles was proposed. The silicate/surfactant rods could spontaneously aggregate and pack into a long-range ordered hexagonal structure. ${ }^{[9]}$ However, subsequent work suggests that the "silicate rod assembly" is not convincing due to the weak self-assembly of long cylindrical micelles. ${ }^{[10]}$

The self-assembly of block copolymers combined with polymers with different chemical properties together creates a strategy for generating scaffolds for the syntheses of porous frameworks. ${ }^{[1 \mathrm{c}, 11]}$ Commercial Pluronic triblock copolymers, such as P123 and P127, usually act as surfactants, but the pore sizes of as-made porous nanomaterials are limited to less than $12 \mathrm{~nm}$ in the absence of micelle swelling agents mainly caused by their small molecular mass. ${ }^{[12]}$ Amphiphilic copolymers (e.g. Polystyrene-block-poly(ethylene oxide) (PEO-b-PS), Polyisoprene-block-Polystyrene-block-poly(ethylene oxide) (PI-PS-PEO), Poly(styrene-block4-vinylpyridine) (P4VP-b-PS), Poly(ethylene oxide)-block-Poly(methyl methacrylate) (PEOb-PMMA)) with high molecular mass have therefore been extensively exploited for the synthesis of wide-pore materials based on spherical micelles or three-dimensional periodic networks. ${ }^{[1 \mathrm{c}, 11 \mathrm{c}]}$ However, morphologically these materials are mainly spherical, bulk, film-like 
or hierarchical. Tubular frameworks with large inner diameter templated by self-assembly of synthesized non-Pluronic amphiphilic block copolymers ${ }^{[3 b, 13]}$ or pluronic triblock copolymers in the presence of pore-expanding agents, are rarely reported. ${ }^{[14]}$

We present here a method for preparing long polymer nanotubes (PNTs) with uniform diameters templated by commercially available cylindrical diblock copolymer micelles. The effect of the reactive conditions, such as template quantity, water/ethanol ratios and initial reactive temperatures, on the framework and morphology of PNTs was investigated. The cylindrical surfactant/precursor composites can be directly transformed to nitrogen-doped carbon nanotubes (NCNTs) through a pyrolysis procedure. NCNTs with high surface area, abundant hierarchical framework structures and plentiful nitrogen doping may provide promising carbon nanomaterials for bioelectrochemistry, and energy conversion and storage. Importantly, it was found that interfacial polymerization and assembly between templates and precursors are complicated and crucial for the composites and structures of the final products. The method can also be used for synthesis of other porous materials, such as silica nanotubes, with desired structure and composition.

\section{Results and Discussion}

PNTs with uniform diameter were synthesized by a templating polycondensation process using 3-aminophenol (AP) and formaldehyde (F) as the monomers, together with diblock copolymer PS-b-PEO (PS187-b-PEO136) surfactant. The fabrication process starts with cylindrical PS-bPEO micelle formation in a mixture of THF, water, and ethanol (Scheme S1 and Figure S1). Auto-catalyzed polycondensation between AP and F takes place on the corona of cylindrical PS-b-PEO micelles, resulting in long cylindrical PS-b-PEO/APF composites. After THF extraction, long polymer nanotubes are formed. Details of the synthesis are given in Table S1.

FESEM and TEM suggest that the PS-b-PEO/APF-5 (Table S1) obtained has a worm-like cylindrical morphology, high aspect ratio, and uniform diameters of $49 \pm 3 \mathrm{~nm}$ (Figure 1a, 1b, 
and Figure S2). After THF extraction, corresponding long PNT-5 with uniform diameter $49 \mathrm{~nm}$ is formed (Figure 1c and 1d). Notably, the ends of part of the PNTs are open, indicating instability of APF resins at the end of the PS-b-PEO/APF composites (Figure 1c and S3). Further, bamboo-joint-like hollow frameworks are observed for PNT-5 because the cylindrical PS-b-PEO micelles are probably first composed from spherical micelles, ${ }^{32}$ the structures of which are frozen during APF resin polymerization.

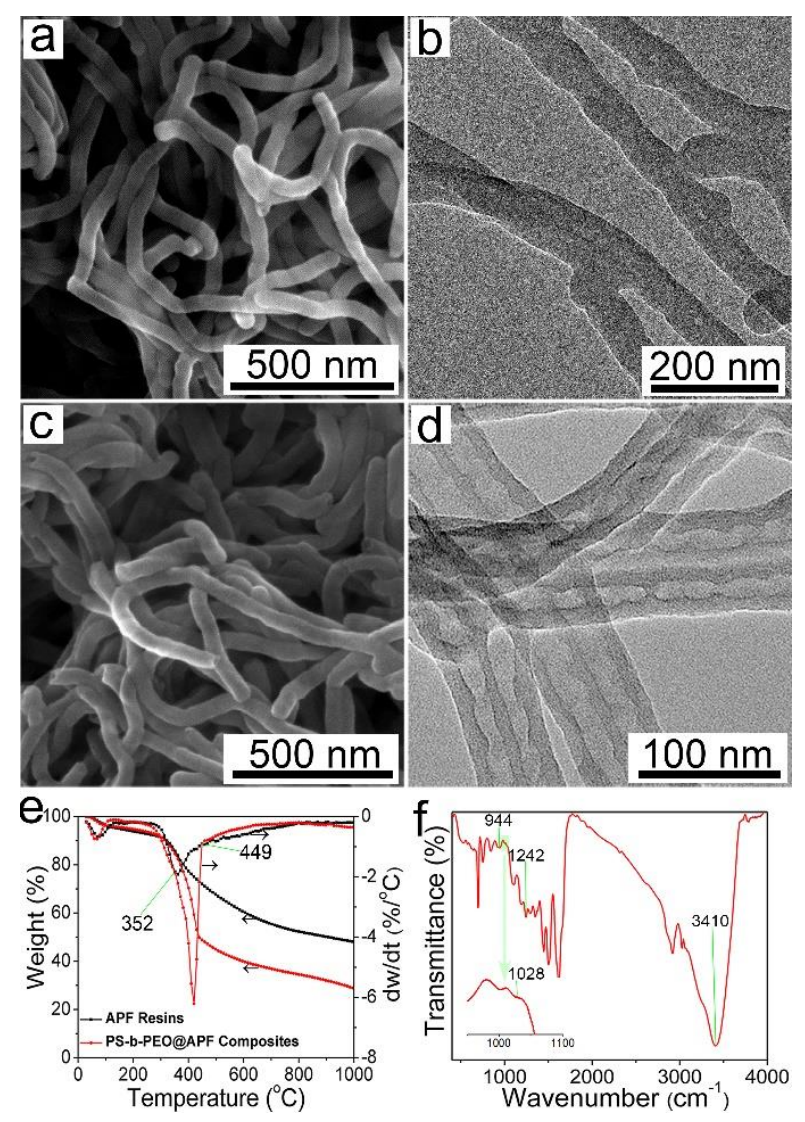

Figure 1. Morphology of cylindrical PS-b-PEO/APF-5, scanning electron microscopy (SEM) image (a) and transmission electron microscopy (TEM) image (b); SEM image (c) and TEM image (d) of PNT-5. (e) TGA and DTG curves for APF resins (black) and PS-b-PEO/APF-5 (red). (f) FTIR spectrum of PS-b-PEO/APF-5.

The control among the polymerization or polycondensation of the precursors, the micellization of surfactants and their assembly is crucial for mesophase formation. ${ }^{[6]}$ The reaction conditions were therefore further explored. In the absence of PS-b-PEO, the product is polydisperse APF polymer microspheres (Figure S4a and S5a). Independent APF polymer 
spheres and some hollow APF polymer spheres adhering on the PNT surface due to the comparatively excess of APF resins (Figure S4b, S5b, S4c and S5c) were observed at low PSb-PEO concentration. The diameters of the PS-b-PEO/APF composites decrease from 275 to $41.2 \mathrm{~nm}$ with increasing amounts of PS-b-PEO from 30 to $270 \mathrm{mg}$ (Figure S4b-h and S5b-h). More cylindrical micelles are formed, resulting in more micelle surface and thinner APF resin walls and thus PS-b-PEO/APF composites and PNTs with smaller diameter. PS-b-PEO is therefore important in the formation of PS-b-PEO/APF composites.

Not only the PS-b-PEO but also the solvent, i.e. water/ethanol volume ratios, affects the morphology of the PS-b-PEO/APF composites and PNTs. In the absence of ethanol, polydisperse cross-linking PS-b-PEO/APF composite spheres are formed (Figure S6a and S7a) and disordered pores in the PNT-10 framework found (Figure S7a). When the water/ethanol ratio changes from $1 / 1$ to $1 / 2$ and 0 , the products are long rods (diameter $41.7 \mathrm{~nm}$ ), to short rods (diameter $41.3 \mathrm{~nm}$ ) and polydisperse cross-linking particles, respectively (Figure S6b-d and S7b-d). The initial reaction temperatures (IRTs) also have a significant effect on the structure of the PS-b-PEO/APF composites and PNTs. The cylindrical morphology can only be formed for IRTs in the range from 0 to $40{ }^{\circ} \mathrm{C}$ (Figure S8a-c and S9a-c). Increasing the IRT to $50{ }^{\circ} \mathrm{C}$ gives PS-b-PEO/APF composites and PNTs with polydisperse cross-linking particles (Figure S8d and S9d). Such morphology of PS-b-PEO/APF composites and PNTs are formed because the reaction conditions can affect the polycondensation of AP and F as well as the micellization of PS-b-PEO, resulting in different product frameworks.

Thermogravimetric analysis (TGA) and derivative thermogravimetry (DTG) were undertaken for PS-b-PEO/APF-5, and of PS-b-PEO/APF-1 as a control, to investigate the thermal stability of PS-b-PEO/APF composites. Figure 1e shows PS-b-PEO/APF-5 with good thermal stability. $\sim 29.2 \%$ of the residual carbon remained even at $1000^{\circ} \mathrm{C}$, caused by the crosslinking structure of resin in the PS-b-PEO/APF-5 skeleton. ${ }^{[16]}$ Comparison of the DTG peaks suggests that the decomposition of PS-b-PEO in PS-b-PEO/APF-5 occurs at temperatures from 
352 to $449{ }^{\circ}{ }^{[17]}$ Therefore, the amount of PS-b-PEO ( 22.4 wt \%) in PS-b-PEO/APF-5 was estimated from the difference between the TGA curves at $450{ }^{\circ} \mathrm{C}$. Fourier transform infrared (FTIR) spectroscopy of PS-b-PEO/APF-5 indicates that the ether stretching peaks at $\sim 1242$ and $1028 \mathrm{~cm}^{-1}$, and the weak band at $\sim 944 \mathrm{~cm}^{-1}$ are assigned to the benzene ring, to which oxazine is bound, ${ }^{[18]}$ demonstrating that benzoxazine residues are present in the PS-b-PEO/APF-5 framework (Figure 1f). The strongest band at $\sim 3410 \mathrm{~cm}^{-1}$ originates from $-\mathrm{OH} \cdots \mathrm{O}$ intra- and intermolecular hydrogen bond stretching, ${ }^{[19]}$ which is partly ascribed to abundant repeat hydroxide groups in the PS-b-PEO skeleton entrapped in the PS-b-PEO/APF-5 framework, serving as cylindrical template. The PS-b-PEO/APF structure thus derives from APF-based polybenzoxazine wrapping cylindrical PS-b-PEO micelles.

Nitrogen-doped carbon nanotubes (NCNTs) with tunable mesostructures and morphologies were generated by pyrolysis of corresponding PS-b-PEO/APF composites (Figure $2 \mathrm{a}, \mathrm{b}$ and Figure S10). The resultant NCNT-5 exhibits a well-defined nanotubular morphology with uniform outer diameter of $41.8 \mathrm{~nm}$ and inner diameter $22.4 \mathrm{~nm}$ (Figure $2 \mathrm{a}$ and $\mathrm{b}$ ), whose narrow inner tube structure is superior to those of previous works. ${ }^{[20]}$ The diameter contraction of carbon nanotubes results from the structure shrinkage during carbonization. Further, the long nanotubular frame is crushed into macaroni-like morphology during the grinding process (Figure 2b). The inner surface of the carbon nanotubes becomes smooth due to limited thermal stability of the APF resins at the inner corona of the PS-b-PEO micelles, especially those formed between two spherical micelles. EDX demonstrates abundant nitrogen and oxygen doping in the matrix of carbon nanotubes (Figure S11). To better understand the NCNT-5 composition, X-ray photoelectron spectroscopy (XPS) was used to analyze functional groups in the carbon nanotube framework. Small amounts of $\mathrm{sp}^{3} \mathrm{C}$ and kinds of nitrogen groups are formed in the carbon matrix (Figure S12). ID/IG intensity ratio in Raman spectrum was found to be 0.93 , illustrating the structural defects and the disorder existing in the carbon framework of NCNT-5 (Figure S13). 

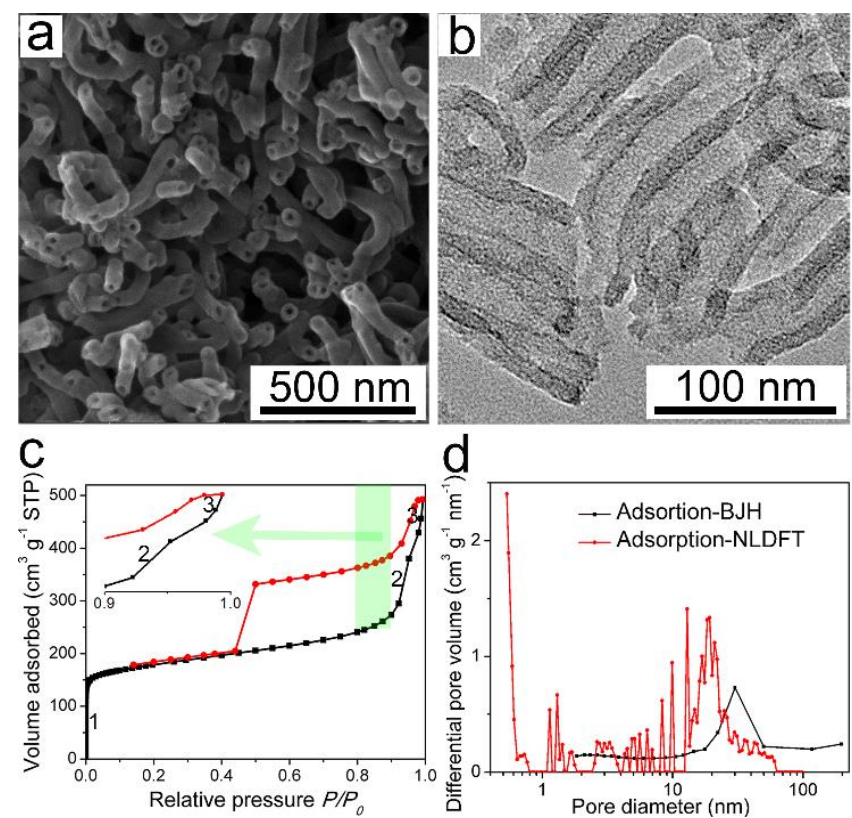

Figure 2. Morphologies of carbon nanotubes: SEM (a) and TEM (b) images. (c) N2 adsorption/desorption isotherms of carbon nanotubes, with an enlarged inset at 0.937 and 0.989 P/P0. (d) Corresponding pore-size distributions according to Barret-Joyner-Halenda (BJH) and nonlocal density functional theory (NLDFT) calculation/simulation.

The nitrogen adsorption/desorption analysis of NCNT-5 shows a typical IV isotherm curve, with three steep condensations on the adsorption part (Figure 2c). The first sharp transition at low pressure results from $\sim 0.5 \mathrm{~nm}$ micropores within carbon nanotubes framework (Figure $2 \mathrm{c}$ and d). According to $\mathrm{BJH}$ theory, the steep condensation 2 at $\sim 0.937 \mathrm{P} / \mathrm{P}_{0}$ corresponds to 30 $\mathrm{nm}$ pores (Figure $2 \mathrm{c}$ and $\mathrm{d}$ ). This value overestimates the cylindrical mesopore diameters because of the inaccuracy of BJH calculations. At higher pressure, a small rise in the isotherm originates from macroporous structures formed through the secondary piled cavity of carbon nanotubes. The BET model was utilized to calculate the surface area of $662 \mathrm{~m}^{2} / \mathrm{g}$ and the pore volume $\mathrm{Vp}=0.76 \mathrm{~cm}^{3} \mathrm{~g}^{-1}$ at $\mathrm{P} / \mathrm{P} 0=0.99$. Moreover, the micropores contribute to most of their specific surface area (SSA), i.e. around 68\%. The other SSA originates from cylindrical tubular mesopores and the tube-piled macropores (Figure S14 and Table S2). The micropores, mesopores and macropores form hierarchical carbon nanotubes, which can facilitate transport 
and storage of ions and/or molecules. NCNTs with high surface area, abundant hierarchical framework structures and plentiful nitrogen doping may provide promising carbon nanomaterials for bioelectrochemistry, and energy conversion and storage. ${ }^{[21]}$

The formation mechanism of the polymer nanotubes or carbon nanotubes includes three main steps (Figure 3): the formation of cylindrical micelles, the polycondensation of APF resins, and extraction of surfactants or carbonization. Diblock copolymer PS-b-PEO probabaly first selfassembles into spherical micelles (step 1a) and is then transformed into long cylindrical micelles as increasing amounts of water and ethanol are added to the PS-b-PEO solution (step 1b). ${ }^{[22]}$ As a consequence, some AP is located in the PEO layers of the PS-b-PEO micelles by strong hydrogen bonds between the hydroxyl and amine groups of AP and the hydroxide groups of the PEO block (step 2a). After adding F solution, the APF oligomers are produced gradually via auto-catalytic polycondensation of 3-aminophenol and formaldehyde. Simultaneously, these oligomers around cylindrical micelles assemble axially along the corona of the cylindrical micelles through strong hydrogen bonding. Continuous polycondensation and cross-linking among 3-aminophenol, formaldehyde, and their oligomers drive the growth of the shell of PSb-PEO/APF composites (step 2b). The cylindrical PS-b-PEO/APF composites are monodisperse without packing into ordered hexagonal mesostructures due to their large diameter and aspect ratio. Finally, the self-assembly process yields a worm-like polymer with radially oriented tubular structures, when PS-b-PEO is extracted (step 3). The carbon nanotubes derived can be obtained by subsequent calcination under argon (step 3). 


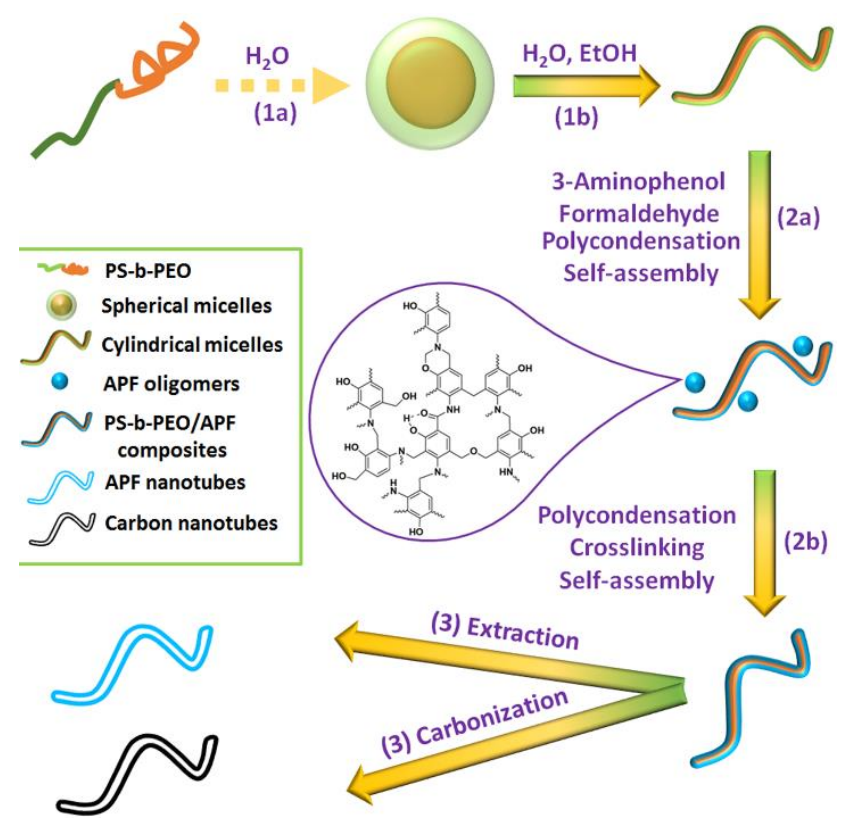

Figure 3. Formation mechanism of the polymer and carbon nanotubes with uniform diameter and large aspect ratio by amphiphilic block copolymer assembly strategy.

Silica nanotubes could be synthesized by a similar process with tetraethyl orthosilicate as precursor. TEM shows that the product has the 1D typically tubular nanostructure, but the length is smaller than that of PNTs (Figure 4). Some silica nanotubes pack into ordered structure within small domains. Although monodisperse nanotubes dominate, some hollow silica nanospheres remain and adhere to the shell of the silica nanotubes. The silica nanotubes have an outer diameter of $40.0 \mathrm{~nm}$, an inner diameter of $27.2 \mathrm{~nm}$, and a wall thickness of $6.8 \mathrm{~nm}$. The synthesis therefore offers a general principle and can probably be extended to other functional nanomaterials, such as polymer, carbon, metal, and oxide nanomaterials.

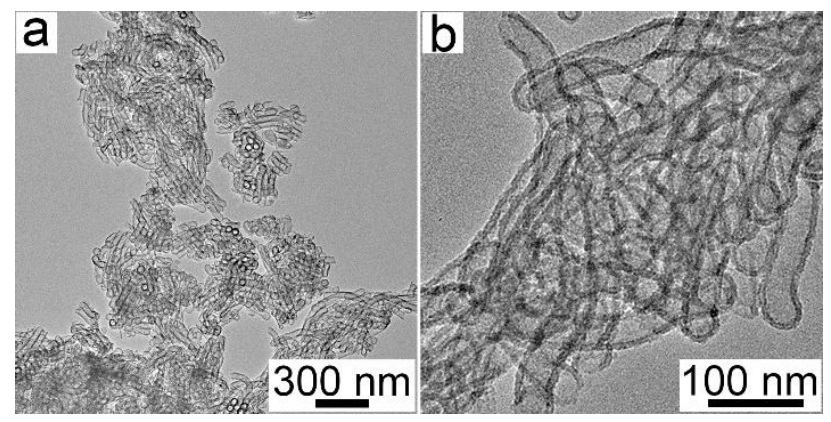

Figure 4. TEM images of the silica nanotubes. 
In general, interfacial polymerization and assembly between templates and precursors are complicated. The self-assembly of templates, the polymerization of precursors, and the interaction between the template micelles and precursors are dominating factors for the morphology and structure of the composite products, further determining the framework and composition of the final porous products. When the template assembles into non-expected micelles (Figure S15a), the precursor forms the corresponding morphology after the polymerization and assembly around the corona of the template micelles (Figure 6d and S7d). When the precursor polymerization of precursors is too strong (Figure S15b), the polymerization process can destroy the structure of the template micelles, leading to the bulk and crosslinking structure despite the presence of expected template micelles (Figure S6a and S7a). When the template frames expected micelles and the interfacial interaction between the micelles and polymerization of precursors is moderate, the precursors can polymerize, assemble and crosslink around the corona of the template micelles. This process decreases the interface between the micelles and precursors, further forming the desired porous nanomaterials after template extraction or thermal treatment.

\section{CONCLUSIONS}

In summary, we have demonstrated a new block copolymer self-assembly strategy to synthesize long polymer nanotubes with large mesochannels. Cylindrical PS-b-PEO micelles serve as the structural template units for composites via self-assembly of PS-b-PEO diblock copolymer, the precursors, and oligomers of APF resins. The structure and chemical composition of the nanotubes can be conveniently modulated by varying the reaction parameters, such as template quantity, water/ethanol ratios and initial reactive temperatures. The corresponding carbon nanotubes are generated by pyrolysis of their organic precursors. Due to the multifarious options of precursors and block copolymers as surfactants, this interfacial interaction-driven approach provides a platform for fabricating a variety of polymers, carbon, metals, oxides and 
their composites with designed structures, functions and properties. These structures and compositions offer promising materials science fields in bioelectrochemistry and energy storage and transfer.

\section{Supporting Information.}

Supporting Information is available from the Wiley Online Library or from the author.

\section{Acknowledgements}

Financial support from the Villum Foundation for a postdoc-program, China Scholarship Council for a PhD scholarship (201706220080), and Fundamental Research Funds of Shandong University (2016JC005, 2017JC042 and 2017JC010) is greatly appreciated.

\section{Conflict of Interest}

The authors declare no conflict of interest.

Keywords: block compolymers, self-assembly, soft-template, mesoporous, nanotubes

[1] a) J. Wei, Z. Sun, W. Luo, Y. Li, A. A. Elzatahry, A. M. Al-Enizi, Y. Deng, D. Zhao, J. Am. Chem. Soc. 2017, 139, 1706; b) T.-Y. Ma, L. Liu, Z.-Y. Yuan, Chem. Soc. Rev. 2013, 42, 3977; c) M. C. Orilall, U. Wiesner, Chem. Soc. Rev. 2011, 40, 520.

[2] C. T. Kresge, M. E. Leonowicz, W. J. Roth, J. C. Vartuli, J. S. Beck, Nature 1992, 359, 710.

[3] a) D. Y. Zhao, J. L. Feng, Q. S. Huo, N. Melosh, G. H. Fredrickson, B. F. Chmelka, G. D. Stucky, Science 1998, 279, 548; b) C. Wang, J. Wei, Q. Yue, W. Luo, Y. Li, M. Wang, Y. Deng, D. Zhao, Angew. Chem. Int. Ed. 2013, 52, 11603.

[4] a) C. D. Liang, K. L. Hong, G. A. Guiochon, J. W. Mays, S. Dai, Angew. Chem. Int. Ed. 2004, 43, 5785; b) W. Luo, T. Zhao, Y. Li, J. Wei, P. Xu, X. Li, Y. Wang, W. Zhang, 
A. A. Elzatahry, A. Alghamdi, Y. Deng, L. Wang, W. Jiang, Y. Liu, B. Kong, D. Zhao, J. Am. Chem. Soc. 2016, 138, 12586; c) Y. Deng, T. Yu, Y. Wan, Y. Shi, Y. Meng, D. Gu, L. Zhang, Y. Huang, C. Liu, X. Wu, D. Zhao, J. Am. Chem. Soc. 2007, 129, 1690.

[5] a) S. C. Warren, L. C. Messina, L. S. Slaughter, M. Kamperman, Q. Zhou, S. M. Gruner, F. J. DiSalvo, U. Wiesner, Science 2008, 320, 1748; b) B. Jiang, C. Li, Ö. Dag, H. Abe, T. Takei, T. Imai, M. S. A. Hossain, M. T. Islam, K. Wood, J. Henzie, Y. Yamauchi, Nat. Commun. 2017, 8, 15581; c) Y. Yamauchi, A. Sugiyama, R. Morimoto, A. Takai, K. Kuroda, Angew. Chem. Int. Ed. 2008, 47, 5371.

[6] P. Yang, D. Zhao, D. I. Margolese, B. F. Chmelka, G. D. Stucky, Nature 1998, 396, 152.

[7] G. S. Attard, J. C. Glyde, C. G. Goltner, Nature 1995, 378, 366.

[8] a) A. Monnier, F. Schüth, Q. Huo, D. Kumar, D. Margolese, R. S. Maxwell, G. D. Stucky, M. Krishnamurty, P. Petroff, A. Firouzi, M. Janicke, B. F. Chmelka, Science 1993, 261, 1299; b) Q. Huo, D. I. Margolese, U. Ciesla, P. Feng, T. E. Gier, P. Sieger, R. Leon, P. M. Petroff, F. Schuth, G. D. Stucky, Nature 1994, 368, 317.

[9] C.-Y. Chen, S.-Q. Xiao, M. E. Davis, Microporous Mater. 1995, 4, 1.

[10] Z. Yuan, W. Zhou, Chem. Phys. Lett. 2001, 333, 427.

[11] a) H.-Y. Hsueh, C.-T. Yao, R.-M. Ho, Chem. Soc. Rev. 2015, 44, 1974; b) Y. Mai, A. Eisenberg, Chem. Soc. Rev. 2012, 41, 5969; c) Y. Deng, J. Wei, Z. Sun, D. Zhao, Chem. Soc. Rev. 2013, 42, 4054; d) Z. Lin, S. Liu, W. Mao, H. Tian, N. Wang, N. Zhang, F. Tian, L. Han, X. Feng, Y. Mai, Angew. Chem. Int. Ed. 2017, 56, 1; e) A. H. Groschel, A. Walther, T. I. Lobling, F. H. Schacher, H. Schmalz, A. H. E. Muller, Nature 2013, 503, 247; f) I. Bita, J. K. W. Yang, Y. S. Jung, C. A. Ross, E. L. Thomas, K. K. Berggren, Science 2008, 321, 939; g) Z. Huang, S.-K. Kang, M. Banno, T. Yamaguchi, D. Lee, C. Seok, E. Yashima, M. Lee, Science 2012, 337, 1521.

[12] M. Kruk, Acc. Chem. Res. 2012, 45, 1678. 
[13] a) W.-J. Zhang, C.-Y. Hong, C.-Y. Pan, J. Mater. Chem. A 2014, 2, 7819; b) M. Müllner, T. Lunkenbein, J. Breu, F. Caruso, A. H. E. Müller, Chem. Mater. 2012, 24, 1802.

[14] a) M. Mandal, M. Kruk, Chem. Mater. 2012, 24, 123; b) G. Farid, M. Kruk, Chem. Mater. 2017, 29, 4675.

[15] P. Bhargava, J. X. Zheng, P. Li, R. P. Quirk, F. W. Harris, S. Z. D. Cheng, Macromolecules 2006, 39, 4880.

[16] a) J. Zhao, W. Niu, L. Zhang, H. Cai, M. Han, Y. Yuan, S. Majeed, S. Anjum, G. Xu, Macromolecules 2013, 46, 140; b) H. Ishida, D. P. Sanders, J. Polym. Sci. Polym. Phys. 2000, 38, 3289.

[17] Y. Deng, T. Yu, Y. Wan, Y. Shi, Y. Meng, D. Gu, L. Zhang, Y. Huang, C. Liu, X. Wu, D. Zhao, J. Am. Chem. Soc. 2007, 129, 1690.

[18] H. Ishida, D. P. Sanders, Polymer 2001, 42, 3115.

[19] a) A. Chernykh, J. Liu, H. Ishida, Polymer 2006, 47, 7664; b) D. J. Allen, H. Ishida, Polymer 2007, 48, 6763; c) H.-D. Kim, H. Ishida, J. Phy. Chem. A 2002, 106, 3271.

[20] a) X. Ao, H. Sun, C. Wang, J. Li, Y. Ruan, B. Li, Q.-H. Wu, Y. Li, J. Jiang, Y. Yang, L. Mai, Carbon 2018, 130, 599; b) S. Zuo, J. Chen, W. Liu, X. Li, Y. Kong, C. Yao, Y. Fu, Carbon 2018, 129, 199.

[21] a) H. Tian, Z. Lin, F. Xu, J. Zheng, X. Zhuang, Y. Mai, X. Feng, Small 2016, 12, 3155; b) H. Tian, S. Zhu, F. Xu, W. Mao, H. Wei, Y. Mai, X. Feng, ACS Appl. Mater. Interfaces 2017, 9, 43975.

[22] D. E. Discher, A. Eisenberg, Science 2002, 297, 967.

Block copolymer self-assembly-induced synthesis of monodisperse mesoporous polymer and nitrogen-doped carbon nanotubes with uniform diameter size and large tubular diameter is proposed for the first time. The method is general and can be extended to prepare other tubular 
nanomaterials, such as silica. Interfacial polymerization and self-assembly among precursors and surfactants are crucial for the morphology, structure and composite of products.

Jianming Zhao, Wei Huang, Pengchao Si, Jens Ulstrup, Fangyuan Diao and Jingdong Zhang* General Syntheses of Nanotubes Induced by Block Copolymer Self-Assembly

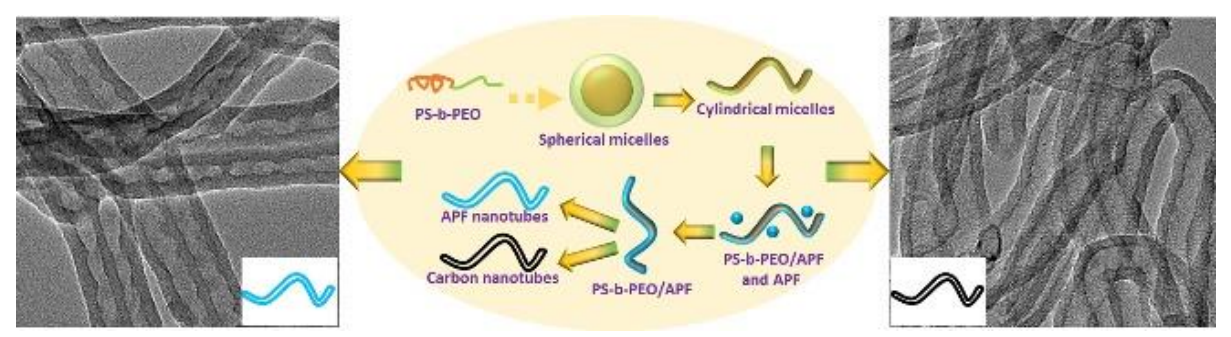

Supporting Information 
for Macromol. Rapid Commun., DOI: 10.1002/marc.2013\#\#\#\#

\section{General Syntheses of Nanotubes Induced by Block Copolymer Self-Assembly}

Jianming Zhao, Wei Huang, Pengchao Si, Jens Ulstrup, Fangyuan Diao and Jingdong Zhang*

Dr Jianming Zhao, Wei Huang, Prof. Jens Ulstrup, Prof. Jingdong Zhang

Department of Chemistry, Technical University of Denmark, Kemitorvet 207, DK-2800

Kongens Lyngby, Denmark.

E-mail: jz@kemi.dtu.dk

Prof. Pengchao Si, Fangyuan Diao

Key Laboratory for Liquid-Solid Structural Evolution and Processing of Materials, Ministry of Education, School of Materials Science and Engineering, Shandong University, Jinan 250061, People's Republic of China.

Keywords: block compolymers, self-assembly, soft-template, mesoporous, nanotubes 


\section{Experimental Section}

\subsection{Chemicals}

Tetraethylorthosilicate (TEOS), 3-Aminophenol (99\%, CAS number: 95-55-6), formaldehyde solution (37 wt. \%, CAS number: 50-00-0), ethanol (> 99.8 \%, CAS number: 64-17-5), tetrahydrofuran (>99.9\%, CAS number: 109-99-9) and hydrochloric acid solution ( $\mathrm{HCl}, 37$ wt \%) were from Sigma. Diblock copolymers PS19.5-b-PEO6 (the number 19.5 means the molecular mass of PS is $19500 \mathrm{Da}$ and the number 6 means the molecular mass of PEO is 6000 Da) was from Polymer Source Inc (Canada). All chemicals were used as received.

\subsection{Preparation of APF resin nanotubes and their corresponding carbon nanotubes}

90 mg of PS-b-PEO (Molecular formula PS187-b-PEO136 is simplified as PS-b-PEO) was first dissolved in $8 \mathrm{~mL}$ tetrahydrofuran (THF), and $24 \mathrm{~mL} \mathrm{H}_{2} \mathrm{O}$, and $12 \mathrm{~mL}$ ethanol then added under vigorous stirring. After stirring for $20 \mathrm{~min}$ at $20^{\circ} \mathrm{C}, 158.2 \mathrm{mg} \mathrm{3}$-aminophenol was dispersed in the mixture. Then, $0.215 \mathrm{ml}$ formaldehyde solution was injected to polymerize with 3aminophenol $30 \mathrm{~min}$ later. The color of the solution became yellow gradually. After continuous reaction and stirring at $20^{\circ} \mathrm{C}$ for $12 \mathrm{~h}$ and then at $70{ }^{\circ} \mathrm{C}$ for $2 \mathrm{~h}$, the yellow solid product was washed by water, centrifugated twice at $10000 \mathrm{rpm}$, and dried at $85^{\circ} \mathrm{C}$ overnight. A THF-aided extraction method was also used to remove the template. For a typical extraction procedure, 10 mg of as-made products was mixed with $1 \mathrm{~mL}$ THF and stirred at room temperature for $24 \mathrm{~h}$. After washing by ethanol and water, polymer nanotubes were obtained. Calcination was carried out in a tubular furnace at $900{ }^{\circ} \mathrm{C}$ for $10 \mathrm{~h}$ under Ar flow. The heating rate was $2{ }^{\circ} \mathrm{C} \mathrm{min}-1$.

Detailed parameters and products for the syntheses are given in table S1.

\subsection{Synthesis of silica nanotubes}

The synthesis was similar to that of NCNT-5, except that tetraethylorthosilicate replaced 3aminophenol and formaldehyde as precursor and heated at relatively low temperature for removing templates without argon protection. Typically, $90 \mathrm{mg}$ of amphiphilic diblock 
copolymer PEO19.5-b-PS6 was dissolved in $8 \mathrm{~mL}$ THF to form a clear solution in a flask, and $24 \mathrm{~mL} \mathrm{H} 2 \mathrm{O}$ and $12 \mathrm{~mL}$ ethanol then added. After stirring for $20 \mathrm{~min}$ at $20{ }^{\circ} \mathrm{C}, 3 \mathrm{ml} 37 \mathrm{wt} \%$ $\mathrm{HCl}$ solution was added to the solution. After $1 \mathrm{~h}, 2.5 \mathrm{ml}$ TEOS was added under stirring at $20^{\circ} \mathrm{C}$. The solution gradually became more and more opaque. After continuous reaction and vigorous stirring for $48 \mathrm{~h}$ at $20^{\circ} \mathrm{C}, 12 \mathrm{~h}$ at $40^{\circ} \mathrm{C}$ and $12 \mathrm{~h} 60^{\circ} \mathrm{C}$, the white solid product was formed and washed by water, centrifugated twice at $10000 \mathrm{rpm}$, and dried at $85^{\circ} \mathrm{C}$ overnight. To prepare silica nanotubes, the cylindrical PS-b-PEO/silica composites were heated at $2{ }^{\circ} \mathrm{C}$ $\min ^{-1}$ from room temperature to $550^{\circ} \mathrm{C}$ and kept at this temperature for $3 \mathrm{~h}$ in air.

\subsection{Materials characterization}

Transmission electron microscopy (TEM) and Energy-Dispersive-X-ray Spectroscopy (EDX) were conducted using a Tecnai T20 G2 electron microscope operated at $200 \mathrm{kV}$. The ground TEM samples were suspended in ethanol and dropped onto a carbon-coated copper grid, followed by drying at room temperature. Field-emission scanning electron microscopy (FESEM) was conducted using a Quanta FEG 200 ESEM system. Nitrogen sorption isotherms were recorded using a Quantachrome Autosorb-1-MP (Quantachrome, USA) at $77 \mathrm{~K}$. Prior to the measurements, the sample was degassed in vacuum at $140{ }^{\circ} \mathrm{C}$ for at least $8 \mathrm{~h}$. The BrunauerEmmett-Teller (BET) method was applied to calculate the specific surface areas using adsorption data in a relative pressure range from 0.05 to 0.25 . The pore size distribution was calculated employing the equilibrium model of non-local density functional theory (NLDFT method) for cylindrical pore geometry (for sample of silica nanotubes) or Barrett-JoynerHalenda (BJH) method (for sample of NCNTs). Total pore volumes were estimated from the adsorbed amount at a relative pressure $\mathrm{P} / \mathrm{P} 0$ of 0.995 . TGA measurements were carried out on a SDTQ600 analyzer from 25 to $1000{ }^{\circ} \mathrm{C}$ under $\mathrm{N} 2$ with a heating rate of $10{ }^{\circ} \mathrm{C} \mathrm{min}^{-1}$. Fourier transform infrared (FTIR) spectra were recorded using a Nicolet Fourier spectrophotometer. Xray photoelectronic spectroscopy (XPS) was carried out at room temperature using a JPS9010TR (JEOL) instrument with an Mg Ka X-ray source. All the binding energies were 
calibrated via referencing to $\mathrm{C} 1 \mathrm{~s}$ binding energy $(284.6 \mathrm{eV})$. Raman spectra were obtained using a Raman spectrometer (Renishaw InVia, $633 \mathrm{~nm}$ Laser).

\section{TEM characterization of PS-b-PEO micelles}

To observe diblock copolymer PS-b-PEO micelle morphologies under TEM with the original morphological sizes and geometries as in the solution, $20 \mu 1$ PS-b-PEO solution (before adding formaldehyde) was "quenched" in $1 \mathrm{ml}$ water, and then dialyzed against water for 1 day, to quickly vitrify the PS blocks into its glassy state. $10 \mu$ quenched solution was then placed on a carbon-coated grid. After 10 minutes, the excess solution was soaked away with filter paper. The grids were dried at room temperature and atmospheric pressure overnight before TEM examination. Since the PS blocks were in their glassy state, the "quenched" morphologies were kept in TEM. ${ }^{[\mathrm{s} 1]}$ 


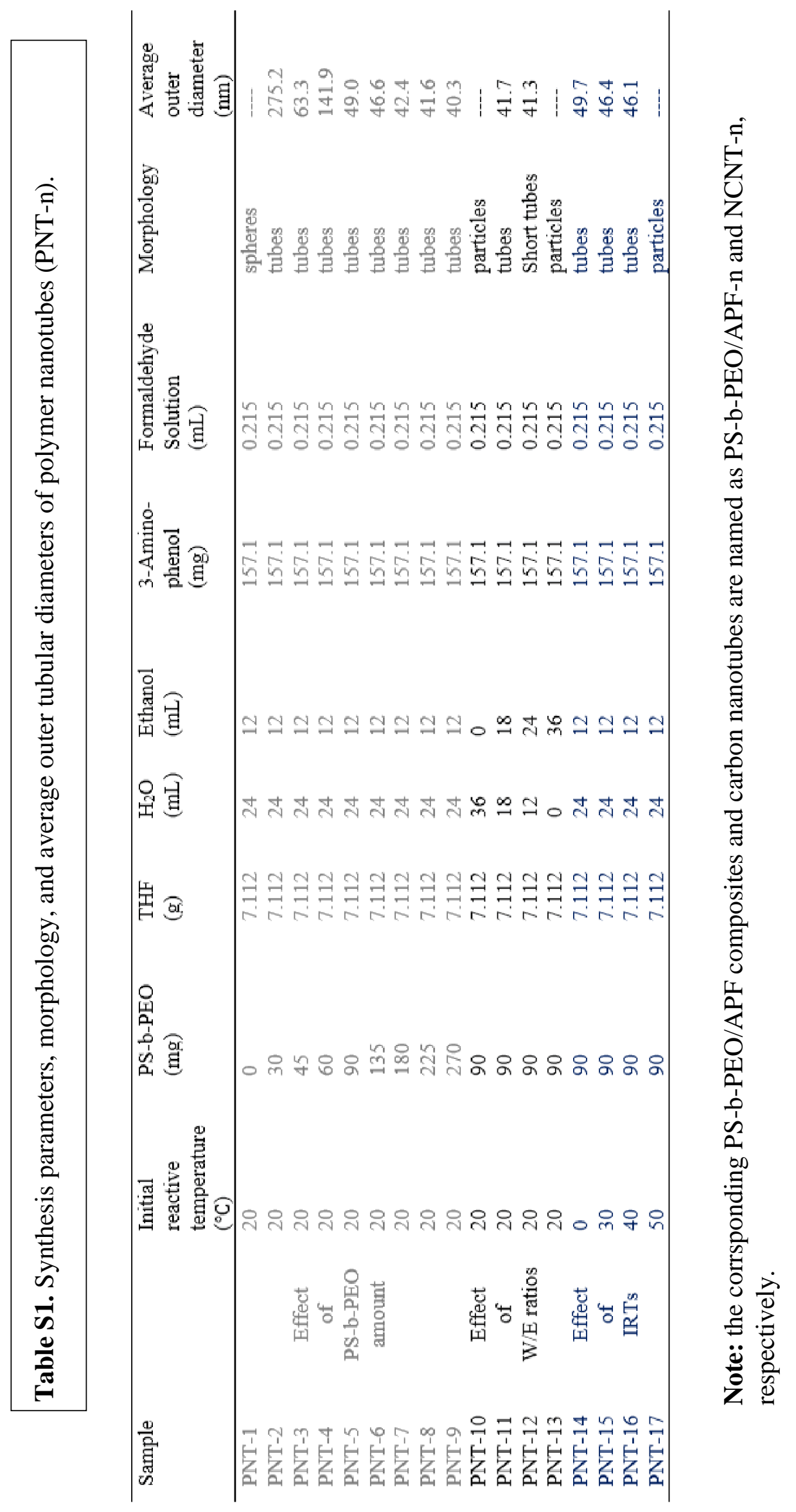




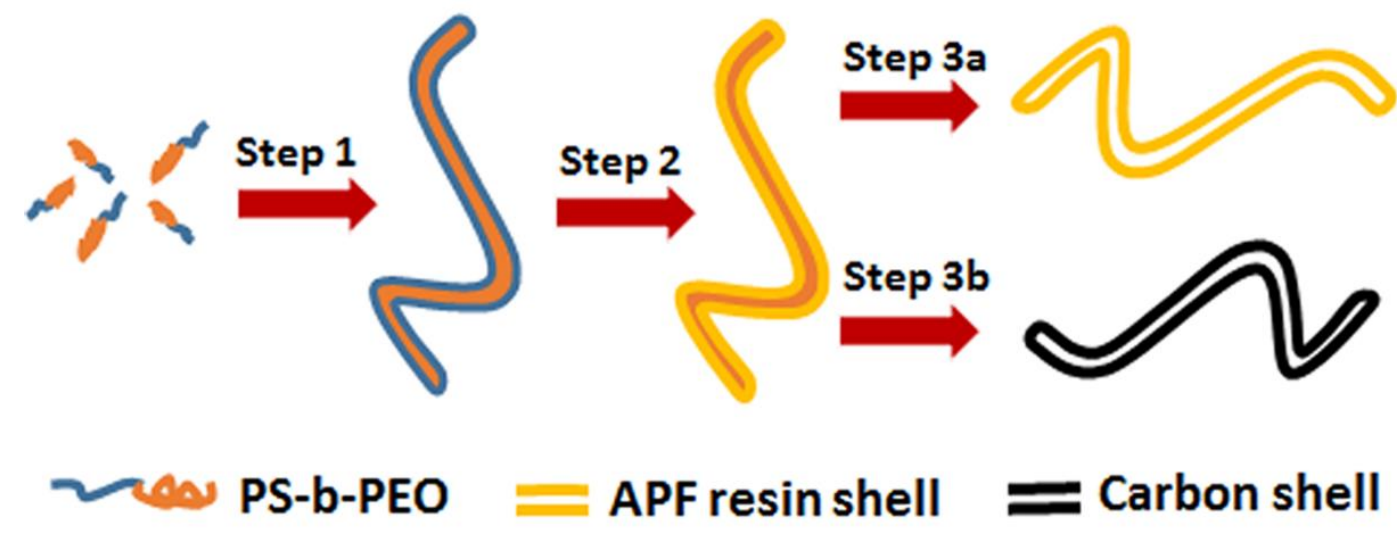

Scheme S1. (a) Schematic illustration of the synthesis process of long APF nanotubes and corresponding carbon nanotubes. Step 1, amphiphilic block copolymer PS-b-PEO selfassembles into long cylindrical micelles driven by shear stress under vigorous stirring. Step 2, formation of long cylindrical PS-b-PEO/APF composites through micelle-induced interface assembly into and on the corona of PS-b-PEO micelles. Step 3a, fabrication of long APF nanotubes with sphere-linking inner mesochannels via extraction treatment of PS-b-PEO/APF composites. Step 3b, synthesis of carbon nanotubes with uniform mesochannels by carbonization of PS-b-PEO/APF composites. 

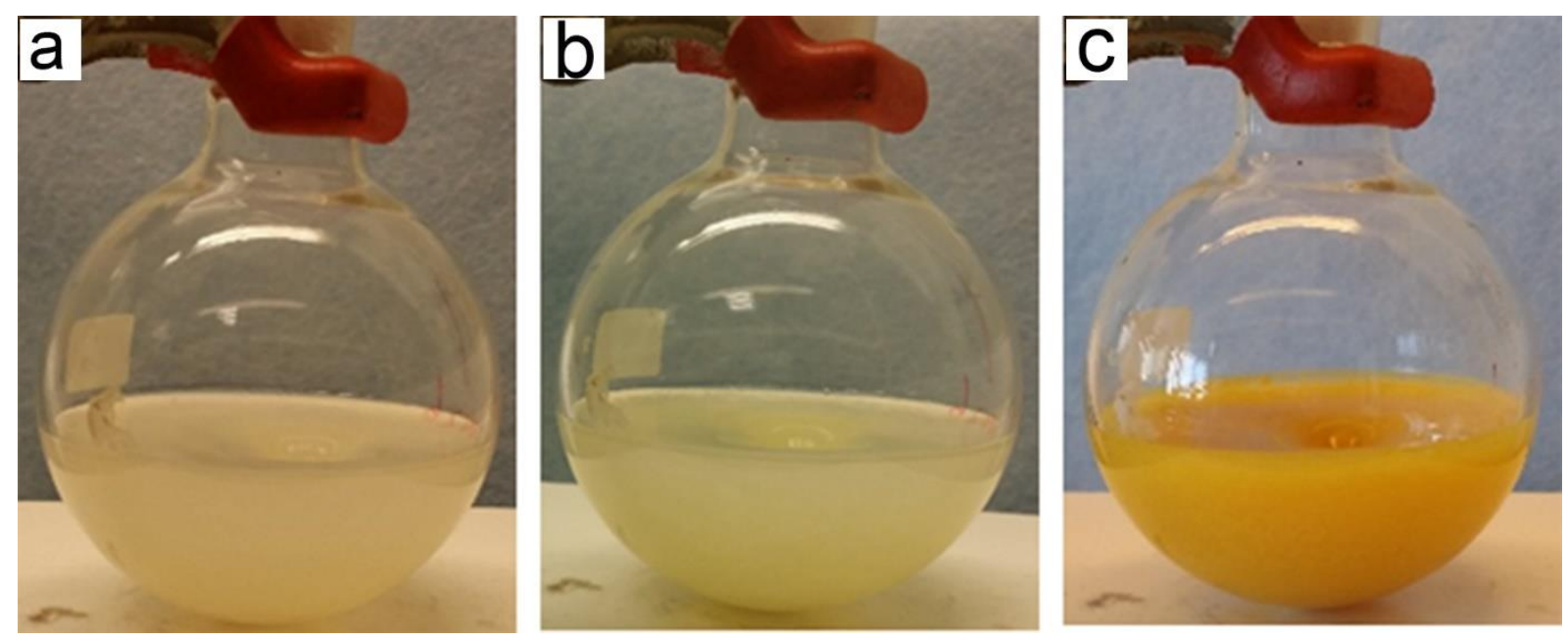

Figure S1. Photographic illustration of the solution color change during the synthesis process. (a) Formation of the cylindrical diblock copolymer PS-b-PEO. (b) Beginning of polymerization of 3-aminophenol and formaldehyde, and assembly to the cylindrical micelles. (c) Further polymerization and crosslinking of 3-aminophenol and formaldehyde, and assembly with the cylindrical micelles. 

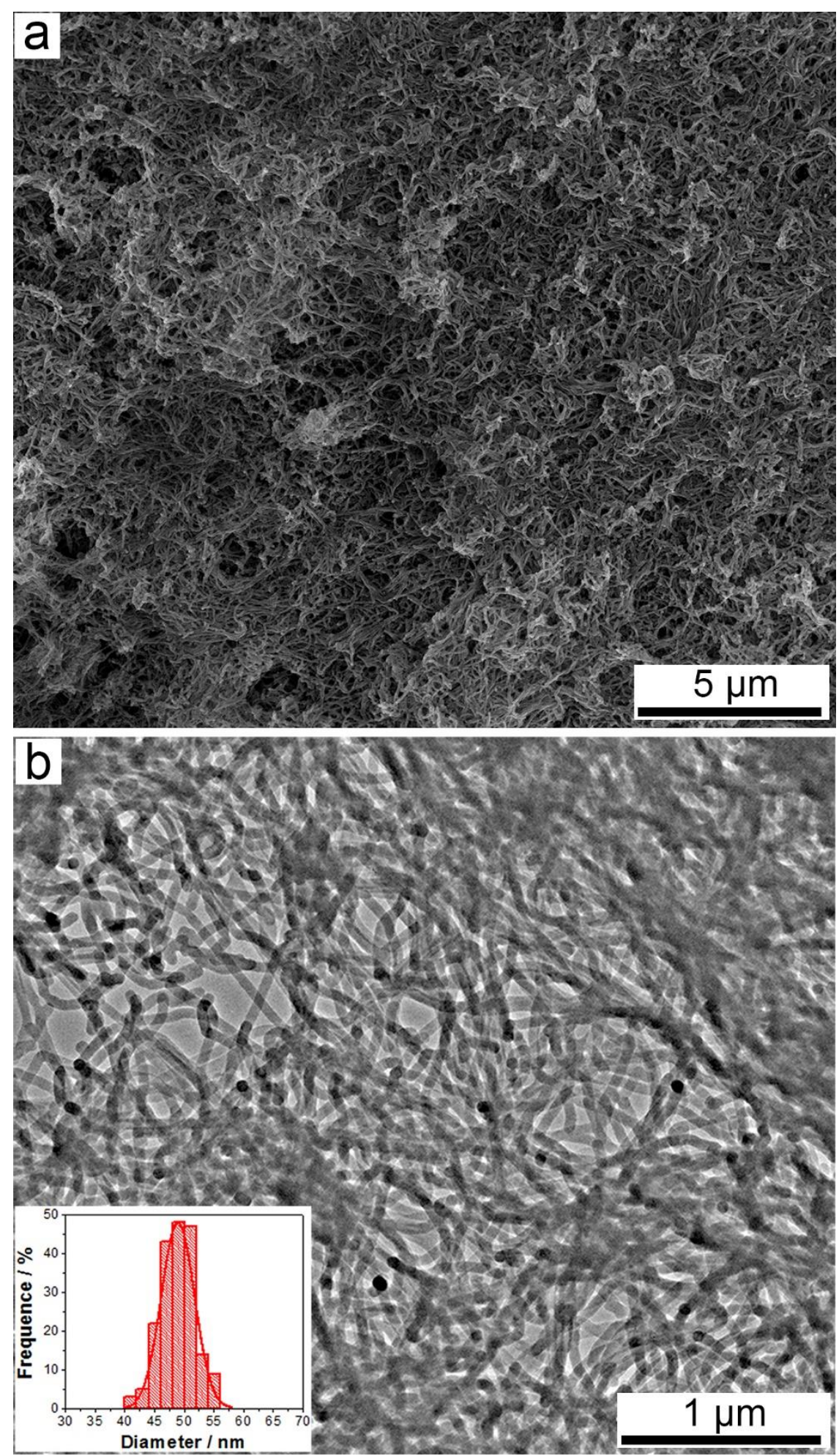

Figure S2. Typical large-scale FESEM (a) and TEM (b) images of PS-b-PEO/APF-5. The inset in Fig. S2b is the diameter distribution histogram of the as-synthesized PS-b-PEO/APF-5. 


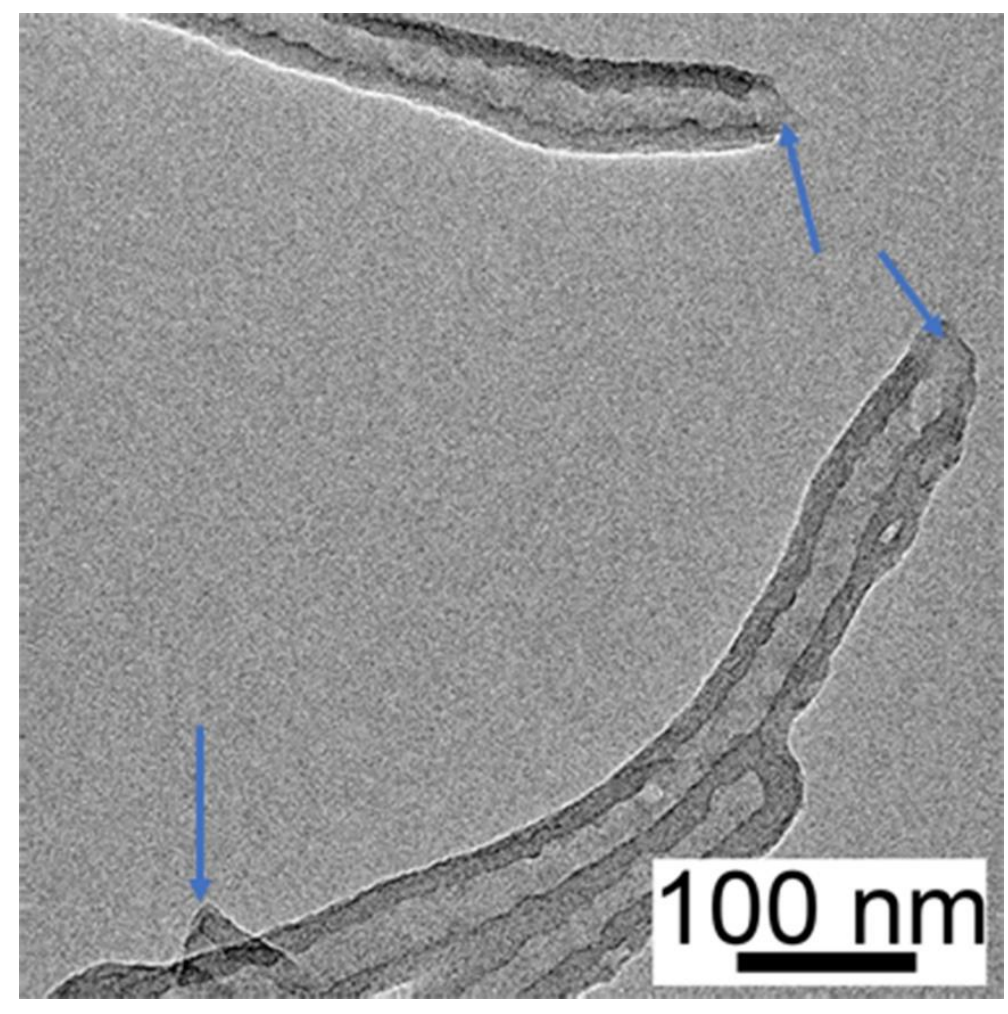

Figure S3. TEM image of PNT-5, blue arrows indicating open ends of PNT-5. 


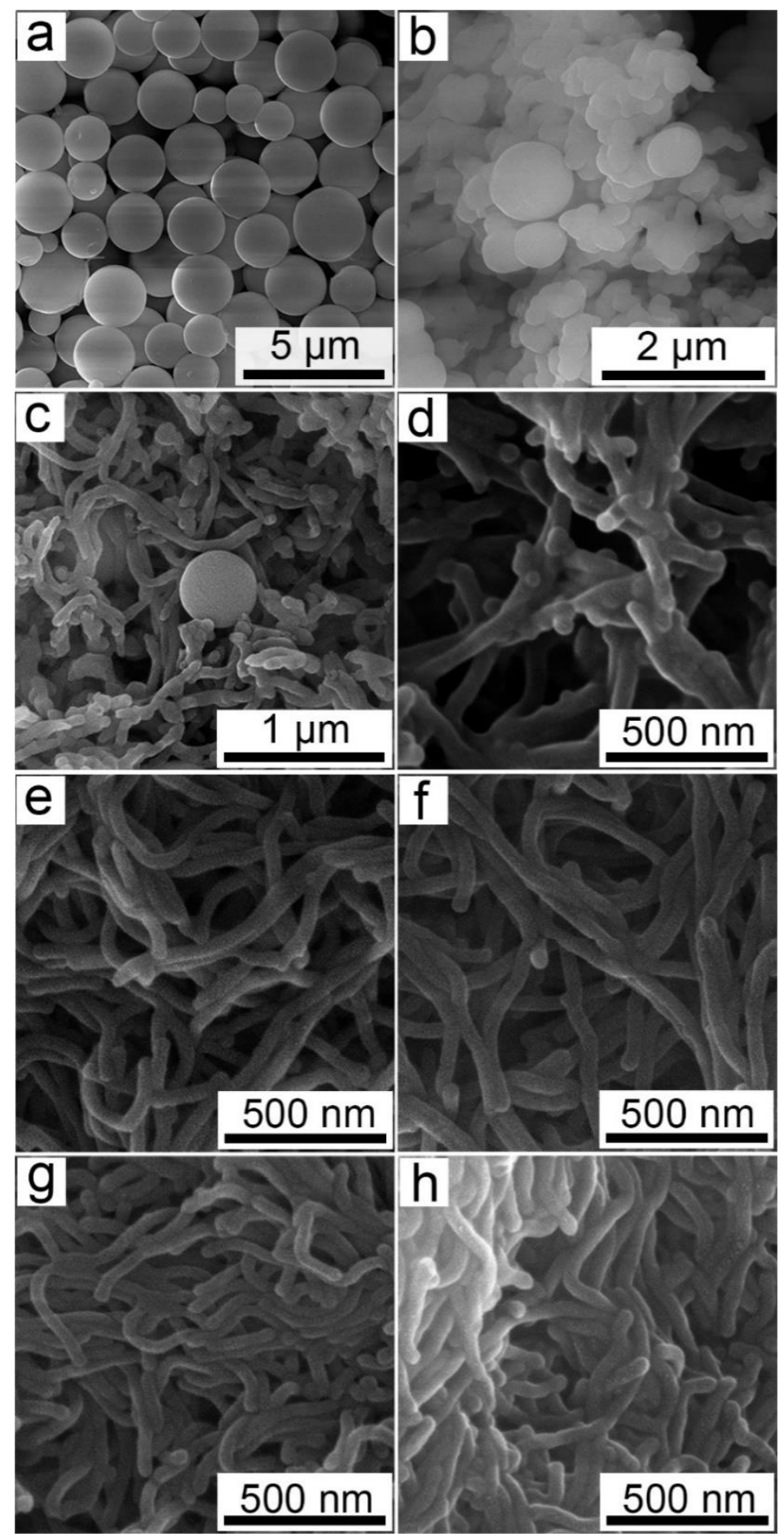

Figure S4. Template effect. FESEM images of PS-b-PEO/APF composites prepared with different quantities of PS-b-PEO: (a) 0 mg (PS-b-PEO/APF-1), (b) 30 mg (PS-b-PEO/APF-2), (c) 45 mg (PS-b-PEO/APF-3), (d) 60 mg (PS-b-PEO/APF-4), (e) 135 mg (PS-b-PEO/APF-6), (f) $180 \mathrm{mg}$ (PS-b-PEO/APF-7), (g) $225 \mathrm{mg}$ (PS-b-PEO/APF-8) and (h) $270 \mathrm{mg}$ (PS-bPEO/APF-9). THF, $7.112 \mathrm{~g}$; water, $24 \mathrm{ml}$; ethanol, $12 \mathrm{ml}$; 3-aminophenol, $157 \mathrm{mg}$; formaldehyde, $215 \mu \mathrm{l}$; initial reaction temperature, $20^{\circ} \mathrm{C}$. 


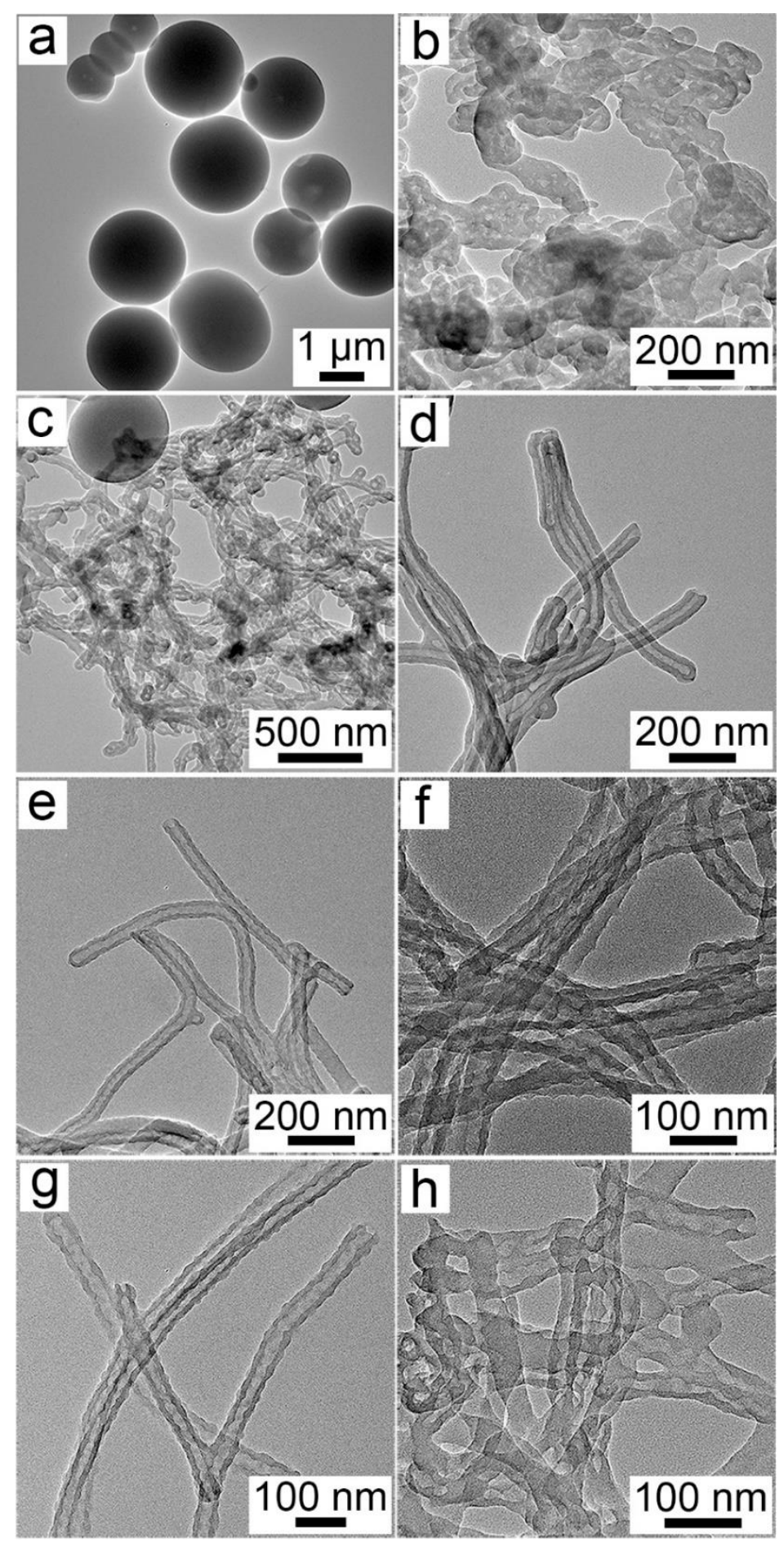

Figure S5. Template effect. TEM images of PNTs prepared from different quantities of PS-bPEO: (a) 0 mg (PNT-1), (b) 30 mg (PNT-2), (c) 45 mg (PNT-3), (d) 60 mg (PNT-4), (e) 135 mg (PNT-6), (f) 180 mg (PNT-7), (g) 225 mg (PNT-8) and (h) 270 mg (PNT-9). THF, 7.112 g; water, $24 \mathrm{ml}$; ethanol, $12 \mathrm{ml}$; 3-aminophenol, $157 \mathrm{mg}$; formaldehyde, $215 \mu \mathrm{l}$; initial reaction temperature, $20^{\circ} \mathrm{C}$. 

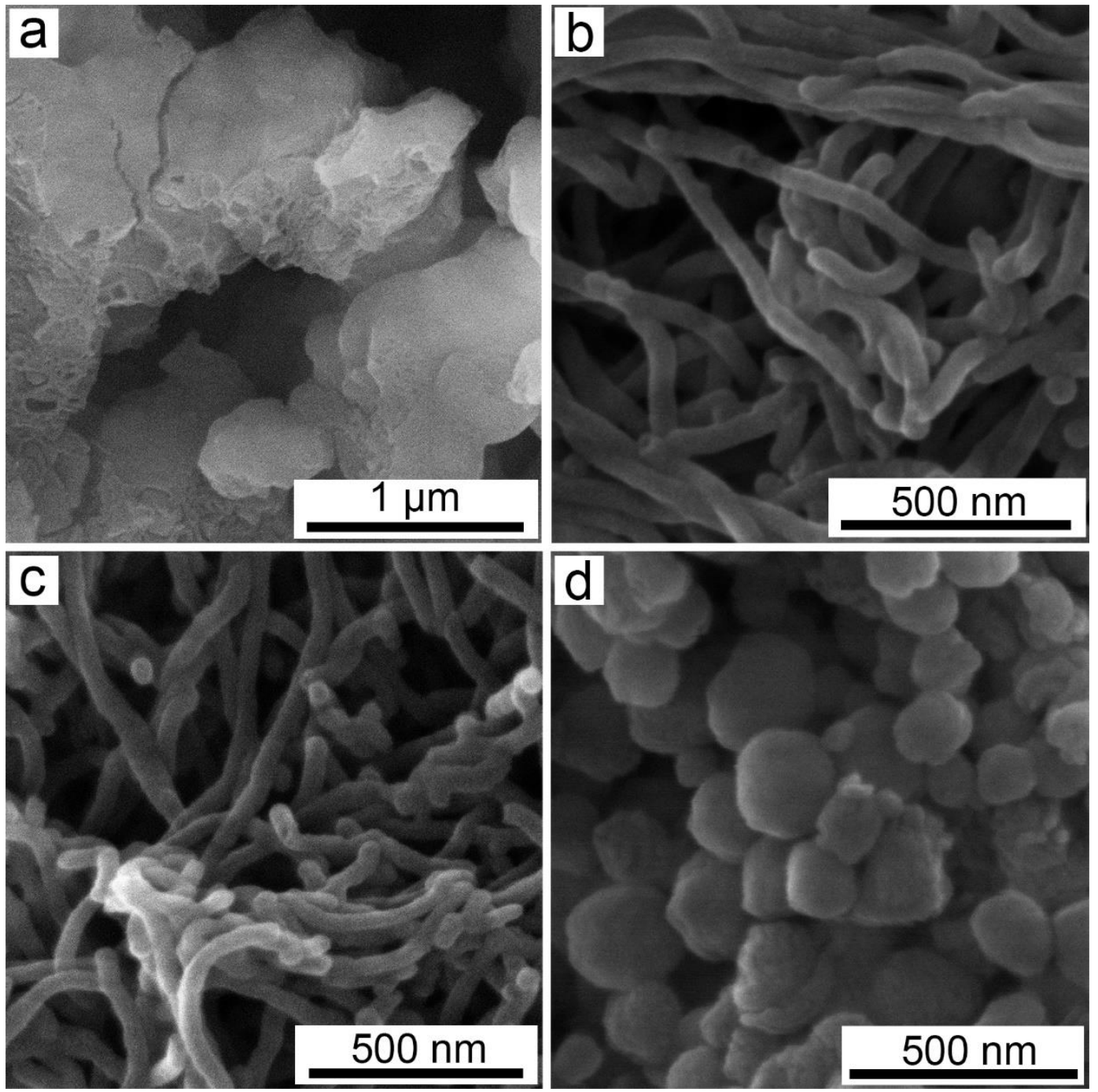

Figure S6. Solvent effect. FESEM images of PS-b-PEO/APF composites prepared at different ratios of water/ethanol (the total volume of water and ethanol is $36 \mathrm{ml}$ ): (a) water (no ethanol) (PS-b-PEO/APF-10), (b) 1/1 (PS-b-PEO/APF-11), (c) 1/2 (PS-b-PEO/APF-12) and (d) 0 (PSb-PEO/APF-13). PS-b-PEO, 90 mg; THF, 7.112 g; 3-aminophenol, $157 \mathrm{mg}$; formaldehyde, 215 $\mu \mathrm{l}$; initial reaction temperature, $20^{\circ} \mathrm{C}$. 


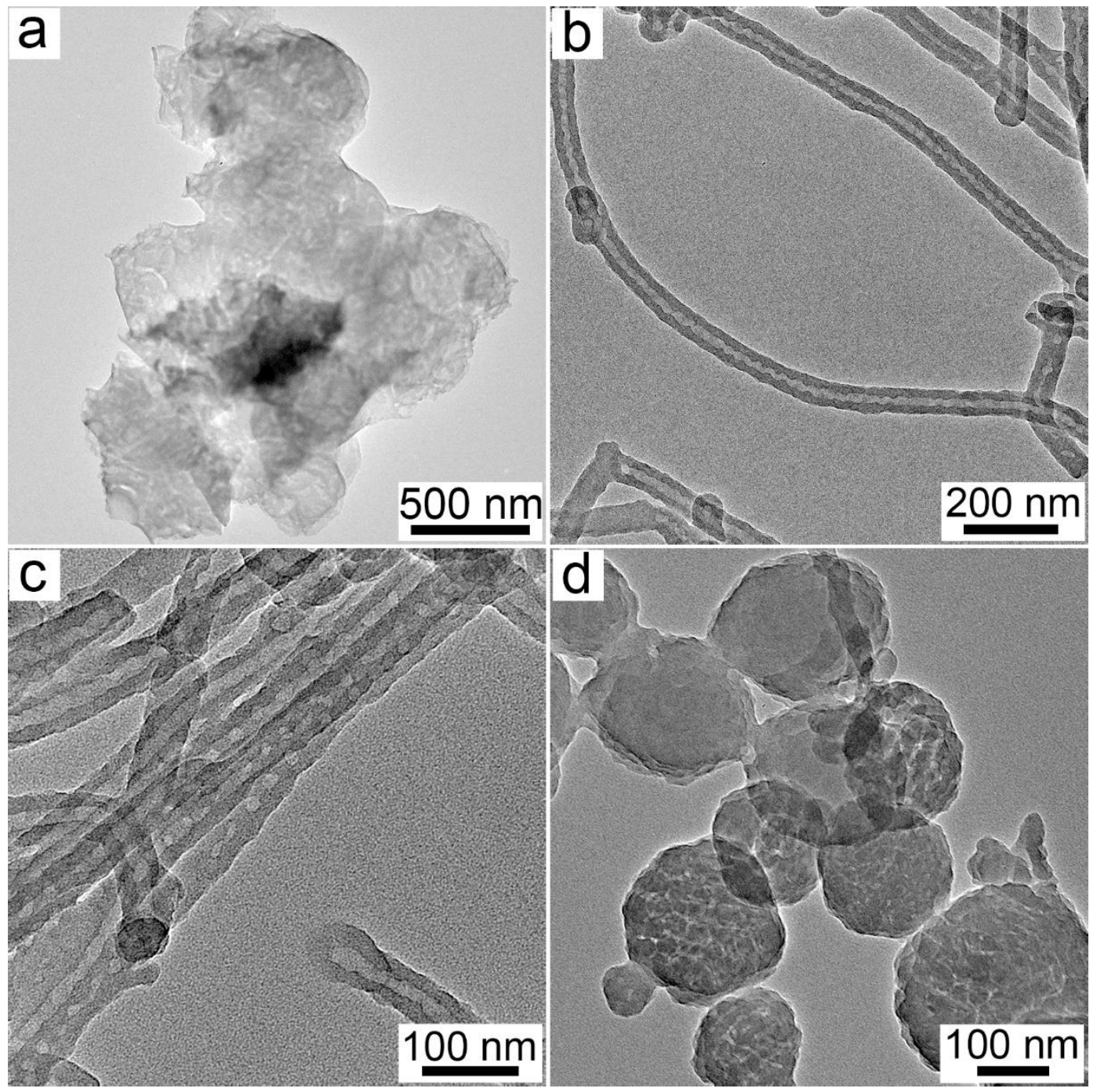

Figure S7. Solvent effect. TEM images of PNTs prepared at different ratios of water/ethanol (the total volume of water and ethanol is $36 \mathrm{ml}$ ): (a) water (no ethanol) (NCNT-10), (b) 1/1 (NCNT-11), (c) 1/2 (NCNT-12) and (d) 0 (NCNT-13). PS-b-PEO, 90 mg; THF, 7.112 g; 3aminophenol, $157 \mathrm{mg}$; formaldehyde, $215 \mu \mathrm{l}$; initial reaction temperature, $20^{\circ} \mathrm{C}$. 


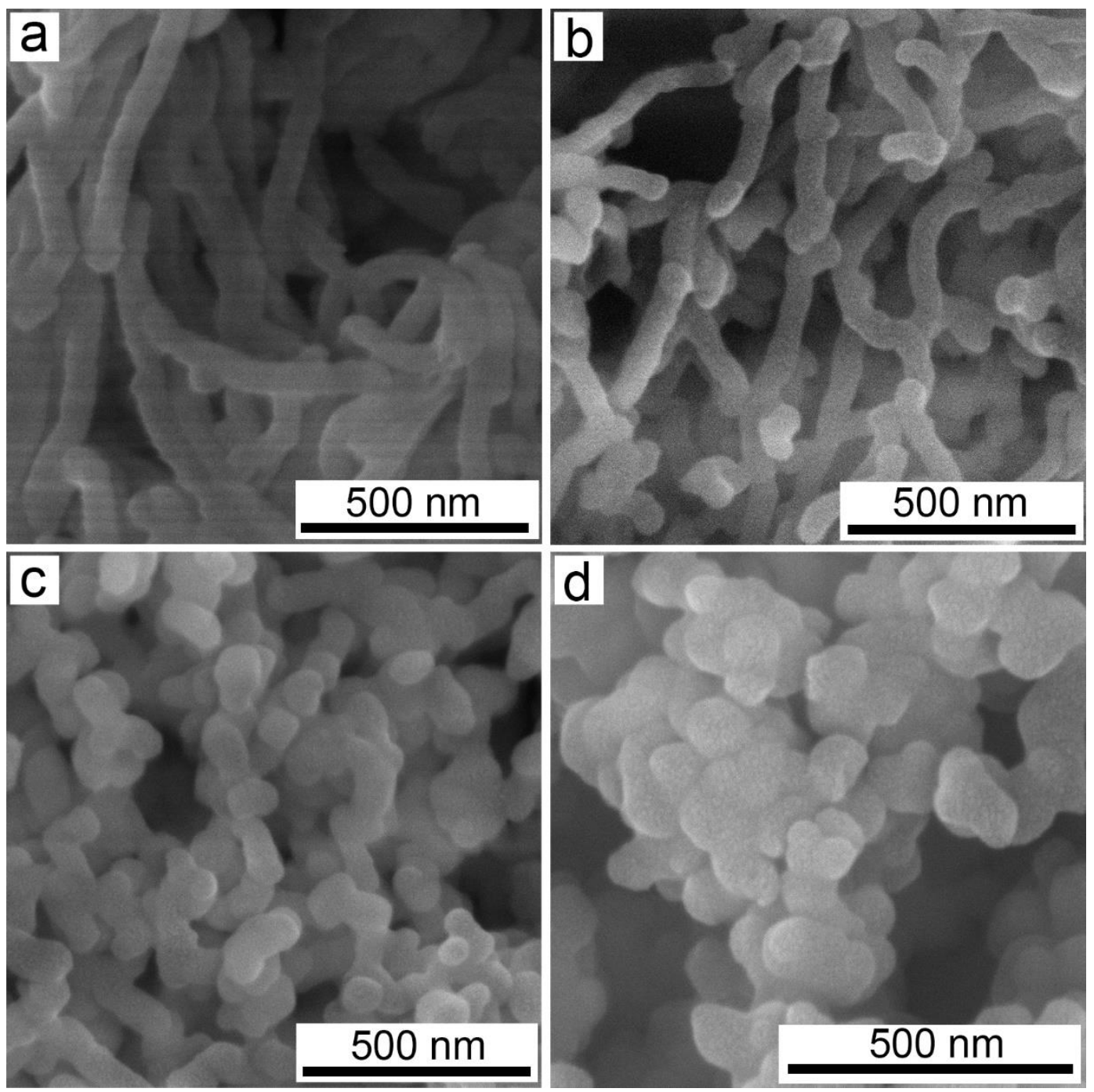

Figure S8. Effect of initial reaction temperature. FESEM images of PS-b-PEO/APF composites prepared at different initial temperatures: (a) $0{ }^{\circ} \mathrm{C}$ (PS-b-PEO/APF-14), (b) $30{ }^{\circ} \mathrm{C}$ (PS-bPEO/APF-15), (c) $40{ }^{\circ} \mathrm{C}$ (PS-b-PEO/APF-16) and (d) $50{ }^{\circ} \mathrm{C}$ (PS-b-PEO/APF-17). PS-b-PEO, $90 \mathrm{mg}$; THF, $7.112 \mathrm{~g}$; water, $24 \mathrm{ml}$; ethanol, $12 \mathrm{ml}$; 3-aminophenol, $157 \mathrm{mg}$; formaldehyde, $215 \mu 1$. 


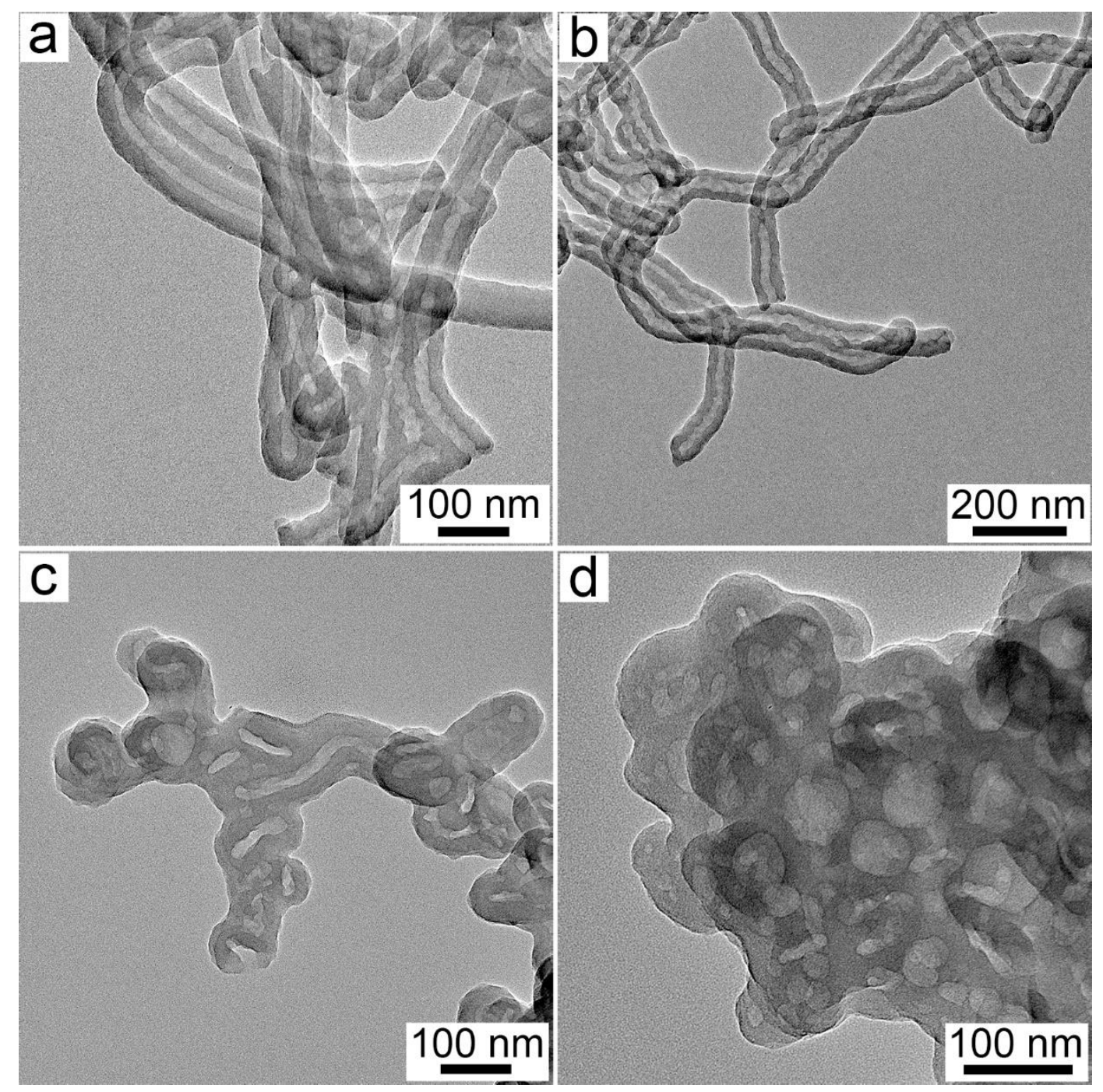

Figure S9. Effect of initial reactive temperature. TEM images of PNTs prepared at different initial temperatures: (a) $0{ }^{\circ} \mathrm{C}$ (NCNT-14), (b) $30{ }^{\circ} \mathrm{C}$ (NCNT-15), (c) $40{ }^{\circ} \mathrm{C}$ (NCNT-16) and (d) $50{ }^{\circ} \mathrm{C}$ (NCNT-17). PS-b-PEO, $90 \mathrm{mg}$; THF, $7.112 \mathrm{~g}$; water, $24 \mathrm{ml}$; ethanol, $12 \mathrm{ml}$; 3aminophenol, $157 \mathrm{mg}$; formaldehyde, $215 \mu \mathrm{l}$. 

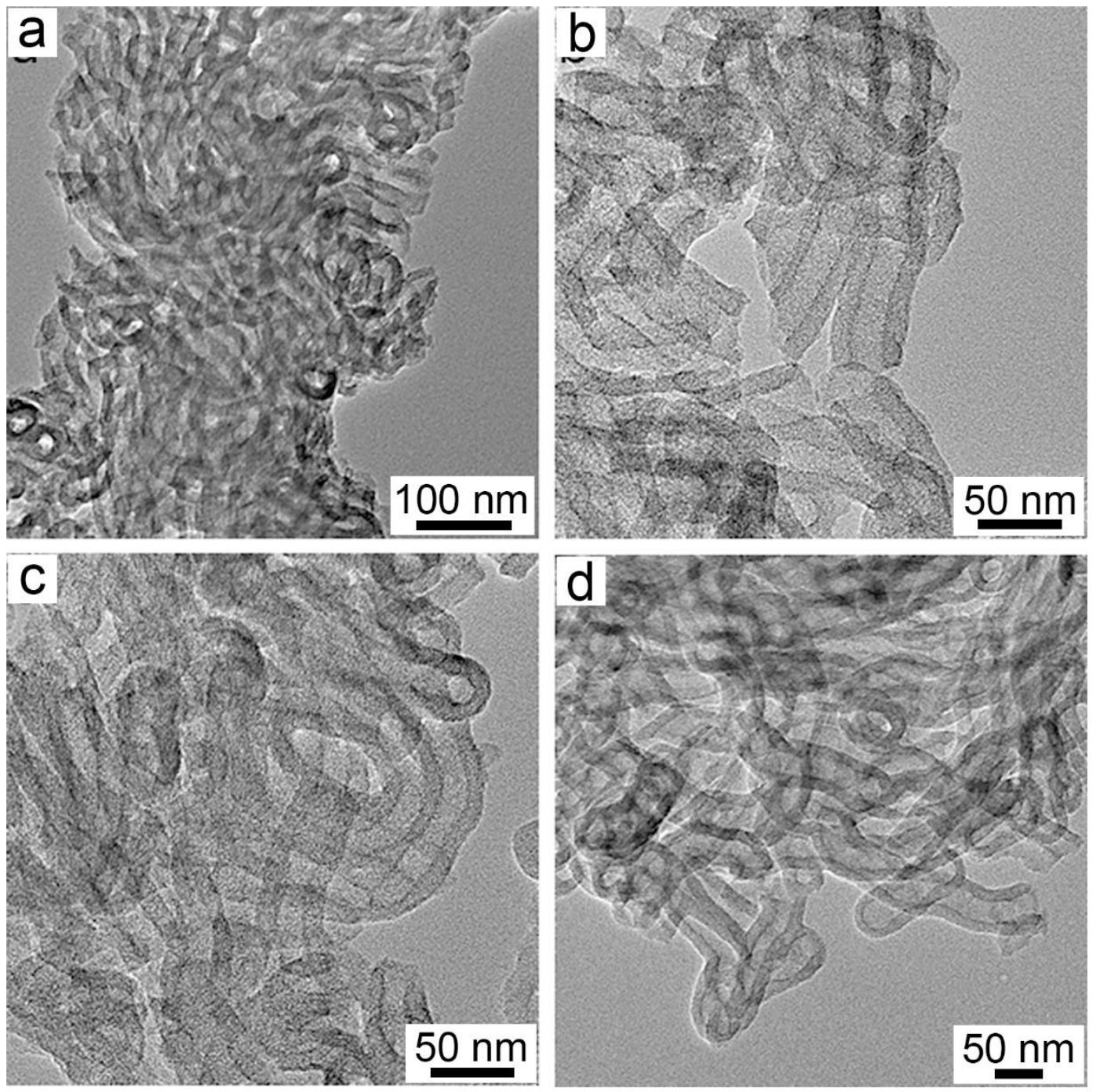

Figure S10. TEM images of (a) NCNT-7, (b) NCNT-11, (c) NCNT-12 and (d) NCNT-15. 

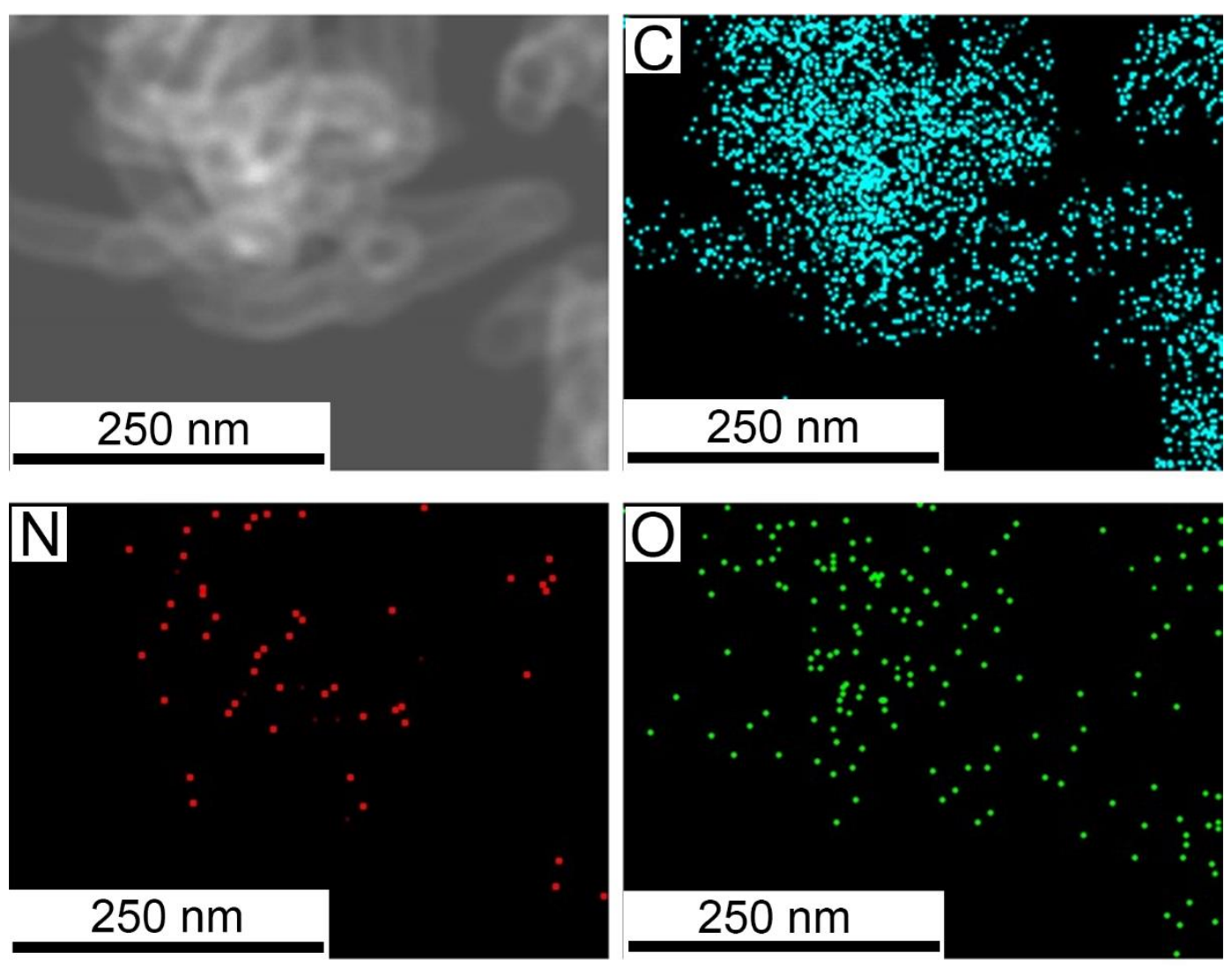

Figure S11. Scanning TEM image and corresponding EDX elemental mapping images of NCNT-5. 

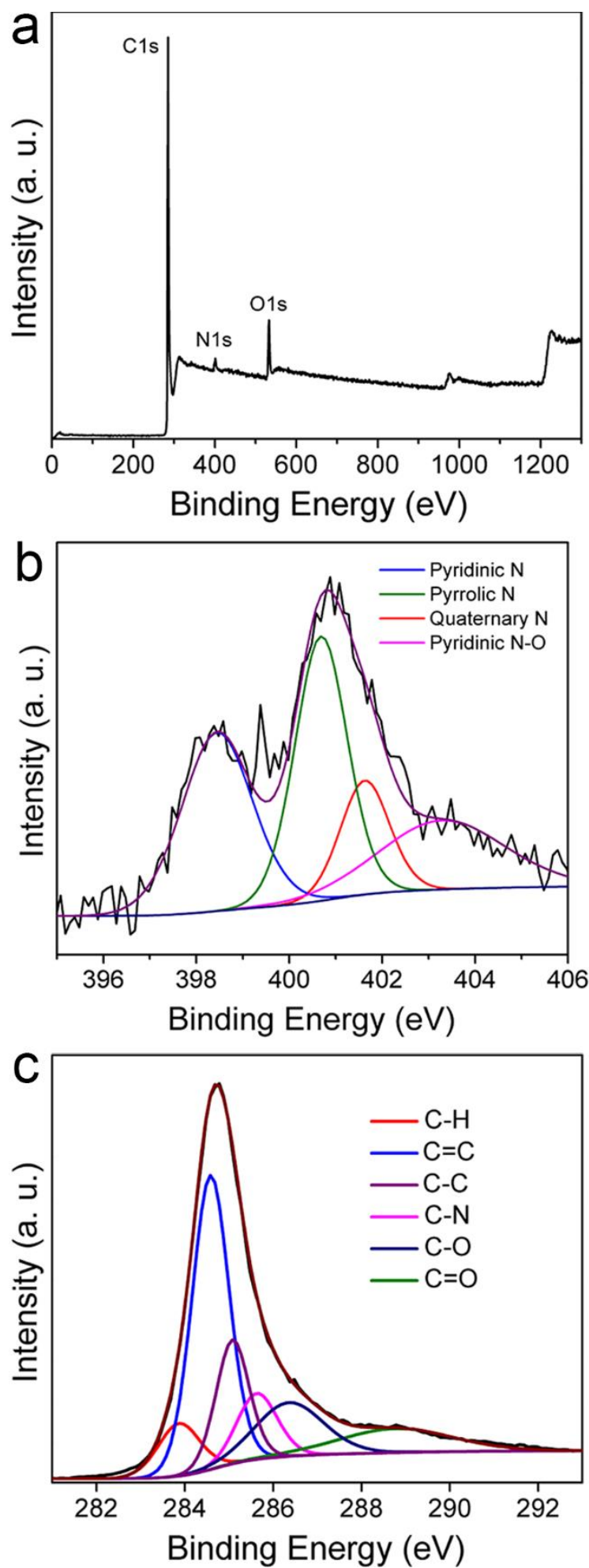

Figure S12. XPS survey spectrum (a) of NCNT-5. High resolution (b) C 1s and (c) N 1s XPS spectra of carbon nanotubes. The contents of $\mathrm{N}, \mathrm{C}$ and $\mathrm{O}$ are $2.30 \%, 90.55 \%$ and $7.15 \%$, respectively. 


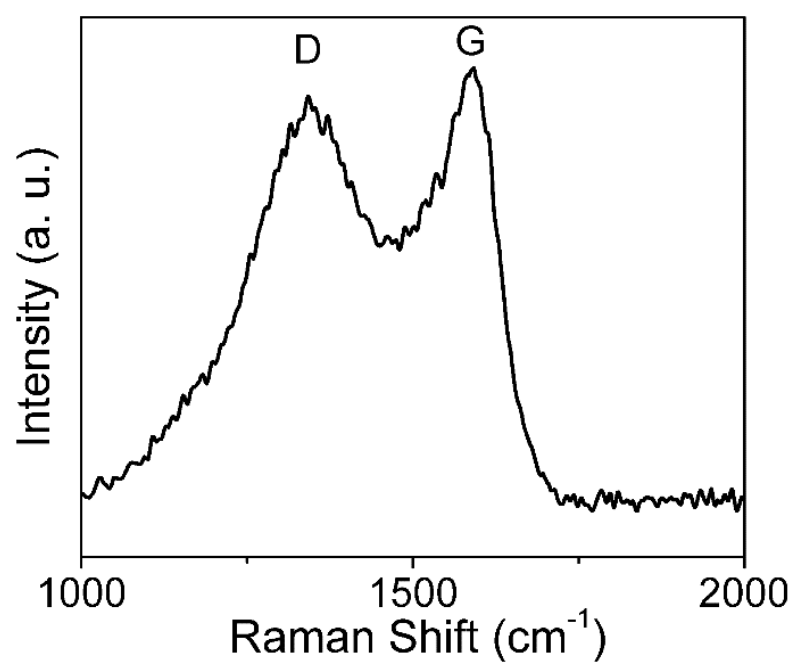

Figure S13. Raman spectrum of NCNT-5. 

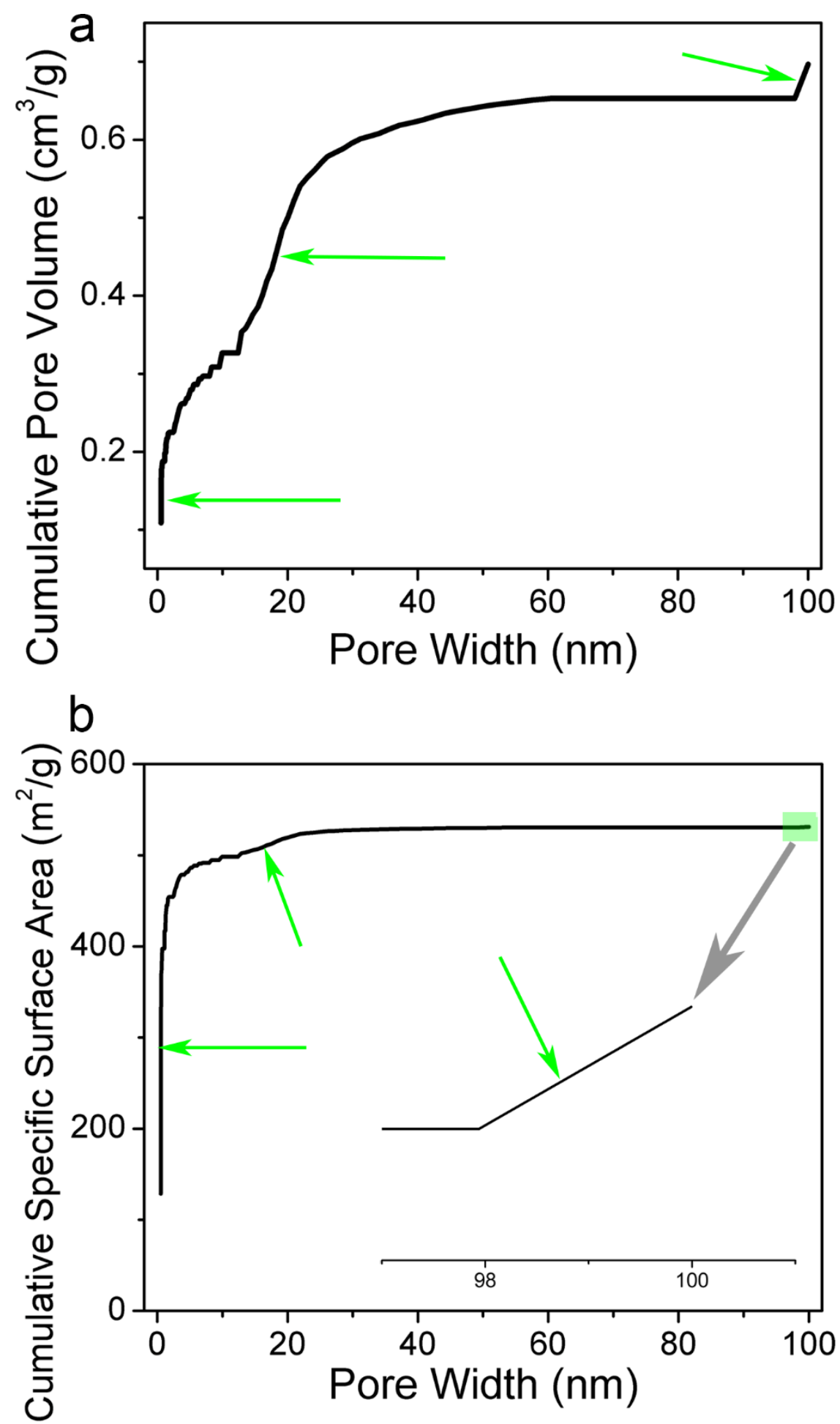

Figure S14. NLDFT cumulative pore volume corresponding to the isotherm of NCNT-5 in Fig. 2c. The three condensations marked reflect the contributions of different-scale "pores" to the specific pore volume (a) and SSA (b) of NCNT-5. Details are given in Supplementary Table S2. Pore volume and SSA data result from the same NCNT-5 topology and thus lead to a similar conclusion. 
Table S2. Partitioned specific volume and SSA according to NLDFT fitting and Fig. S12.

\begin{tabular}{llll}
\hline Condensations & Pore Width $(\mathbf{n m})$ & Volume $\left(\mathbf{c m}^{\mathbf{3}} / \mathbf{g}\right)$ & SSA $\left(\mathbf{m}^{2} / \mathbf{g}\right)$ \\
\hline $\mathbf{1}$ & $<2.0$ & 0.21 & 447 \\
$\mathbf{2}$ & $2.0-50$ & 0.44 & 84 \\
$\mathbf{3}$ & $>50$ & 0.11 & 131 \\
Total & - & 0.76 & 662 \\
\hline
\end{tabular}



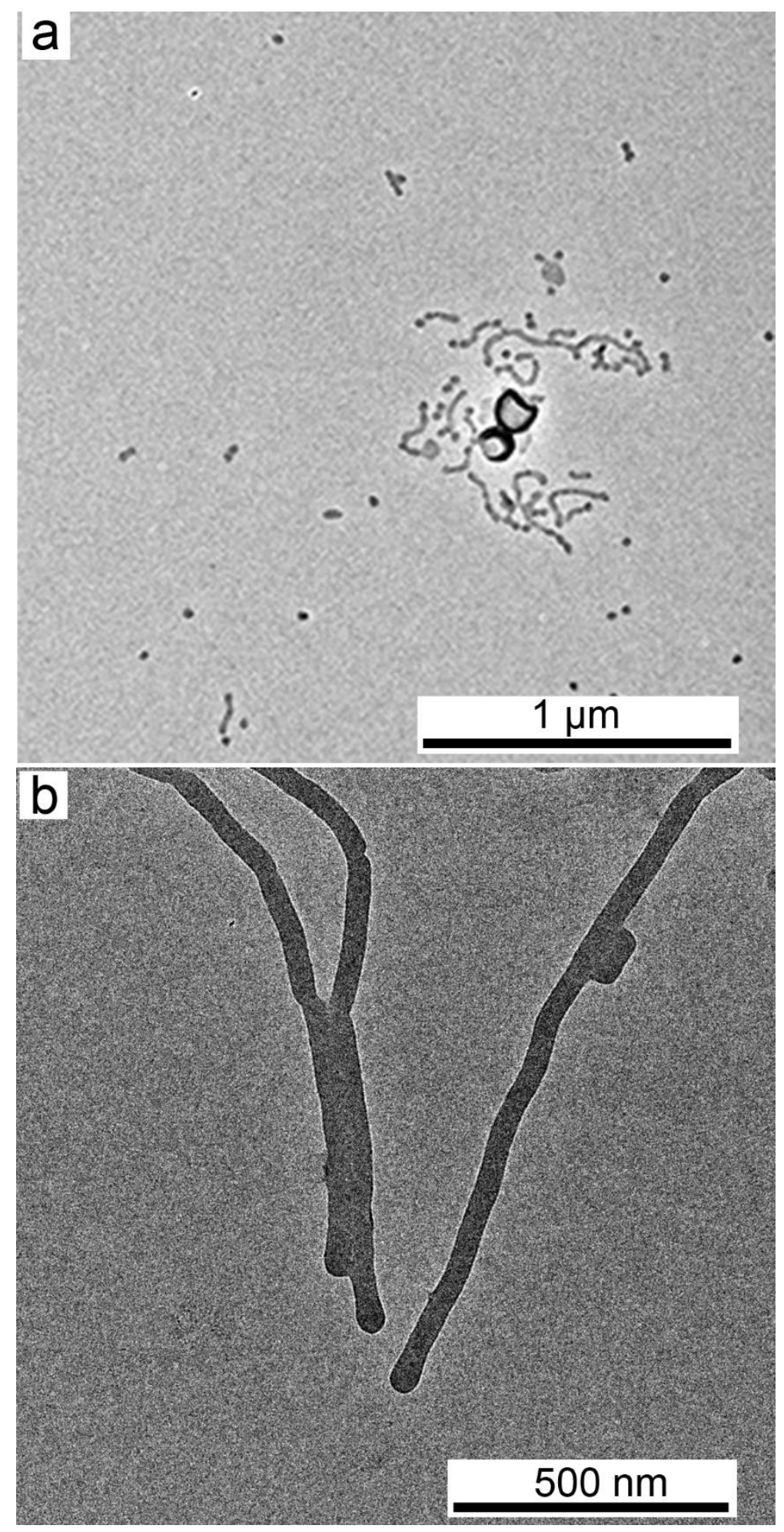

Figure S15. TEM images of PS-b-PEO micelles of (a) PNT-13 and (b) PNT-10. 


\section{Reference}

[s1] Z. Lin, S. Liu, W. Mao, H. Tian, N. Wang, N. Zhang, F. Tian, L. Han, X. Feng, Y. Mai, Angew. Chem. Int. Ed. 2017, 56, 7135. 